\title{
Pointwise gradient bounds for entire solutions of elliptic equations with non-standard growth conditions and general nonlinearities
}

\author{
Cecilia Cavaterra $^{(1,5)} \quad$ Serena Dipierro $^{(2)} \quad$ Alberto Farina $^{(3)} \quad \mathrm{Zu} \mathrm{Gao}{ }^{(4)}$ \\ Enrico Valdinoci ${ }^{(2,1)}$
}

March 13, 2019

(1) - Dipartimento di Matematica "Federigo Enriques"

Università degli studi di Milano

Via Saldini 50, I-20133 Milano (Italy)

(2) - Department of Mathematics and Statistics

University of Western Australia

35 Stirling Highway, WA6009 Crawley (Australia)

(3) - LAMFA, CNRS UMR 7352

Faculté des Sciences

Université de Picardie Jules Verne

33 rue Saint Leu, 80039 Amiens CEDEX 1 (France)

(4) - School of Mathematics and Statistics

Central South University

932 Lushan S Road

410083 Hunan, Changsha (China)

(5) - Istituto di Matematica Applicata e Tecnologie Informatiche "Enrico Magenes", CNR

Via Ferrata 1, 27100 Pavia (Italy)

E-mail addresses: cecilia.cavaterra@unimi.it, serena.dipierro@uwa.edu.au, alberto.farina@u-picardie.fr, gaozu7@163.com, enrico.valdinoci@uwa.edu.au

\begin{abstract}
We give pointwise gradient bounds for solutions of (possibly non-uniformly) elliptic partial differential equations in the entire Euclidean space.

The operator taken into account is very general and comprises also the singular and degenerate nonlinear case with non-standard growth conditions. The sourcing term is also allowed to have a very general form, depending on the space variables, on the solution itself, on its gradient, and possibly on higher order derivatives if additional structural conditions are satisfied.
\end{abstract}

2000 Mathematics Subject Classification: 35J60, 35J70, 35J75.

Keywords: Regularity theory, singular and degenerate equations, $(p, q)$-Laplacian, pointwise gradient estimates in terms of a potential function. 


\section{Introduction}

In this paper we consider a very general elliptic equation, set in the whole of the Euclidean space, and we will establish pointwise gradient bounds for the solutions. The operator taken into account can be degenerate and singular, and we can also consider the case of the superposition of differential operators with different homogeneity.

The main result establishes that (a possibly nonlinear function of) the gradient of the solution is bounded at any point by a suitable potential function. Moreover, the bound obtained, which can be seen as a generalization of the Energy Conservation Principle to PDEs, is in general sharp, since if equality is attained in this bound, the solution is shown to be necessarily constant.

Our results comprise, as particular cases, the classical results in Mod85, CGS94. The method of proof is based on Maximum Principles and it can be seen as a refinement of the classical Bernstein method introduced in [Ber27], as extended in [Pay76, PP80, Spe81]. Namely, one considers a suitable auxiliary function, called "P-function" in jargon, which is defined in terms of the solution and its gradient, and shows that such a $P$-function satisfies a differential inequality: from this and the Maximum Principle, the desired bounds on the gradient plainly follow.

In spite of its intrinsic simplicity (and unquestionable beauty), the idea of obtaining gradient bounds via the Maximum Principle turned out to be very effective, and it found several applications in many topics, including Riemannian geometry (see e.g. [CY75, Ham93, SZ06, FV11]) anisotropic or nonhomogeneous equations (see e.g. [DG02, BG13, FV14, CFV14, BG15, BG15b]), and also subelliptic equations (see Gar09]), and, when the equation is set in a domain, the technique also detects the geometry of the domain itself (see e.g. [FV10b, CFV12]). Moreover, a novel approach to the Maximum Principle method has been recently exploited in a very successful way in [AC13, And15, AX18, in order to obtain oscillation and modulus of continuity estimates. In general, these types of gradient and continuity estimates are also related to rigidity results for overdetermined problems (see e.g. GL89, FV10, FV13b]) and they also provide, as a byproduct, new classification results of Liouville type (see also GS99, PQS07).

In the case under consideration in this paper, given the very general structure of the equation, one needs to exploit a technique introduced in [FV13]: in our case, such differential inequality will be satisfied, in general, only up to a reminder, which can be shown to have the appropriate sign in a number of concrete examples.

Let us now describe in detail the mathematical framework in which we work. We consider the following PDE in divergence form:

$$
\operatorname{div}\left(\Phi^{\prime}\left(|\nabla u|^{2}\right) \nabla u\right)=f(u)+g(\nabla u, S u) \quad \text { in } \mathbb{R}^{n},
$$

where $\Phi \in C_{\text {loc }}^{3, \alpha}((0,+\infty)) \cap C([0,+\infty))$ for some $\alpha \in(0,1)$, with $\Phi(0)=0, f \in C^{1}(\mathbb{R})$ and $g \in$ $C^{1}\left(\mathbb{R}^{n} \times \mathbb{R}^{N-n}\right) \cap L^{\infty}\left(\mathbb{R}^{n} \times \mathbb{R}^{N-n}\right)$.

We denote by $(\zeta, \eta) \in \mathbb{R}^{n} \times \mathbb{R}^{N-n}$ the variables of the function $g$, i.e., $g:=g(\zeta, \eta)$, and we assume that for all $M>0$ we have that

$$
\sup _{\substack{(\zeta, \eta) \in \mathbb{R}^{n} \times \mathbb{R}^{N-n} \\|\zeta| \leq M}}\left|g_{\zeta}(\zeta, \eta)\right|<+\infty, \quad \text { for all } j \in\{1, \ldots, n\},
$$

where the subscript $\zeta_{j}$ denotes partial derivative with respect to the variable $\zeta_{j}$.

In (1.1) and throughout this article,

$$
S: L^{\infty}\left(\mathbb{R}^{n}\right) \cap C^{\ell}\left(\mathbb{R}^{n}\right) \mapsto\left(C^{\ell^{\prime}}\left(\mathbb{R}^{n}\right)\right)^{N-n}
$$


will denote an operator 11 acting on bounded and smooth functions, with $\ell \in[3,+\infty]$ and $\ell^{\prime} \in[1,+\infty]$, and we will write $S=\left(S^{[1]}, \ldots, S^{[N-n]}\right)$ where $N \geqslant n \geqslant 1$. If $N-n=0$, we have that $g$ does not depend on the variable $\eta$.

We stress that $S$ is just a map sending functions into vectorial functions, and it does not necessarily need to be linear or continuous in any topology. Also, for the sake of simplicity, we will consider smooth 2 solutions $u \in C^{\ell}\left(\mathbb{R}^{n}\right)$ of (1.1).

As customary, we will assume that the divergence form operator in (1.1) possesses suitable (possibly singular or degenerate) elliptic structure, which will ensure the validity of the Maximum Principle. For this, for any $\sigma \in \mathbb{R}^{n}$, we set

$$
a_{i j}(\sigma):=2 \Phi^{\prime \prime}\left(|\sigma|^{2}\right) \sigma_{i} \sigma_{j}+\Phi^{\prime}\left(|\sigma|^{2}\right) \delta_{i j},
$$

and we will always assume in this paper that at least one of the following Assumptions A and B is satisfied:

Assumption A. There exist $p>1, a \geq 0$ and $C_{1}, C_{2}>0$ such that, for every $\sigma, \xi \in \mathbb{R}^{n} \backslash\{0\}$,

$$
C_{1}(a+|\sigma|)^{p-2} \leq \Phi^{\prime}\left(|\sigma|^{2}\right) \leq C_{2}(a+|\sigma|)^{p-2}
$$

and

$$
C_{1}(a+|\sigma|)^{p-2}|\xi|^{2} \leq \sum_{i, j=1}^{n} a_{i j}(\sigma) \xi_{i} \xi_{j} \leq C_{2}(a+|\sigma|)^{p-2}|\xi|^{2}
$$

Assumption B. We have that $\Phi \in C^{1}([0,+\infty))$, and there exist $C_{1}, C_{2}>0$ such that, for every $\sigma \in$ $\mathbb{R}^{n}$ and every $\xi^{\prime}=\left(\xi, \xi_{n+1}\right) \in \mathbb{R}^{n} \times \mathbb{R}$, with $\xi^{\prime} \cdot(-\sigma, 1)=0$,

$$
\begin{aligned}
& C_{1}(1+|\sigma|)^{-1} \leq \Phi^{\prime}\left(|\sigma|^{2}\right) \leq C_{2}(1+|\sigma|)^{-1} \\
& C_{1}(1+|\sigma|)^{-1}\left|\xi^{\prime}\right|^{2} \leq \sum_{i, j=1}^{n} a_{i j}(\sigma) \xi_{i} \xi_{j} \leq C_{2}(1+|\sigma|)^{-1}\left|\xi^{\prime}\right|^{2} .
\end{aligned}
$$$$
\text { and }
$$

Related structural assumptions on the diffusive operators have been considered in [CGS94, FV13]. We observe that Assumptions $\mathrm{A}$ and $\mathrm{B}$ will be enforced with $\sigma:=\nabla u$, hence, under a Lipschitz condition on the solution $u$, one has that $|\nabla u| \leq M$ for some $M>0$. So that it will be sufficient to require Assumptions $\mathrm{A}$ and $\mathrm{B}$ with $\sigma$ belonging to the ball of radius $M$ centered at the origin, which we will denote by $B_{M}$. Therefore, from now on, when we say that Assumptions A and B are satisfied, we mean that they are fulfilled when $\sigma \in B_{M}$, and the constants $C_{1}$ and $C_{2}$ can depend

\footnotetext{
${ }^{1}$ For instance, in our setting,
}

$$
S(u)=\left(x, u, x+\nabla u, u_{111}-u_{22}+\Delta^{2} u, u_{11}^{5}, x \cdot \nabla u+\log \left(1+u_{2222}^{4}\right)+\sqrt{-\Delta}(\arctan u)\right)
$$

is an admissible (though not specially meaningful) operator. In this case, $N-n=n+1+n+3$, that is $N=3 n+4$. In our setting, it is an interesting feature that the nonlinear source $g$ can also depend on higher derivatives, on nonlinear differential operators, on integro-differential operators, etc.

${ }^{2}$ In this paper, we did not optimize the regularity assumptions on the solution $u$. For our purposes, it is sufficient to have sufficient regularity to write (1.1) in the pointwise sense and consider its derivatives. Hence, if the operator $S$ only involves a finite number of derivatives, then also $u$ is required to have a finite number of derivatives. When $S$ only involves operators of order 1 or less, in concrete cases one can also apply standard elliptic regularity theory to obtain the desired regularity of $u$ starting with rather minimal assumptions. Since the minimal regularity assumptions in this general setting are rather technical, we will not introduce this additional complication in this article, sticking to the case of sufficiently smooth solutions. 
on $M$. In particular, when Assumption $\mathrm{B}$ is in force, we can reduce to Assumption A with $p=2$, with constants depending on $M$.

In our setting, we have that Assumptions A and B are satisfied by very general nonlinear operators, as established by the following result:

Proposition 1.1. Let $m \geq 1$ and

$$
\Phi(r):=\sum_{k=1}^{m}\left(\frac{2 c_{k}}{p_{k}}\left(b_{k}+r\right)^{\frac{p_{k}}{2}}-\frac{2 c_{k} b_{k}^{\frac{p_{k}}{2}}}{p_{k}}\right),
$$

with

$$
1 \leq p_{1} \leq \ldots \leq p_{m}<+\infty
$$

and

$$
c_{k}>0, \text { for every } k \in\{1, \ldots, m\} \text {. }
$$

Then:

(i) If

$$
\begin{array}{ll} 
& p_{1}>1, \quad b_{1} \geq 0 \\
\text { and } \quad \mu b_{1} \leq b_{k} \leq \frac{b_{1}}{\mu}, \quad \text { for all } k \in\{1, \ldots, m\}
\end{array}
$$

for some $\mu \in(0,1)$, then Assumption A holds true.

(ii) If

$$
\mu \leq b_{k} \leq \frac{1}{\mu}, \quad \text { for all } k \in\{1, \ldots, m\},
$$

for some $\mu \in(0,1)$, then Assumption B holds true.

In view of Proposition 1.1 it follows that Assumptions A and B comprise the important case of nonlinear operators with non-standard growth conditions and with non-uniform ellipticity properties, see [AM01, CMM17, BCM18].

In our setting, the bounds on the gradient of the solution $u$ will require the control on the sign of a suitable reminder. To describe this feature in details, we give some notation. For any $r \in \mathbb{R}$, we define

$$
\Lambda(r):=2 r \Phi^{\prime \prime}(r)+\Phi^{\prime}(r) .
$$


In this setting, the reminder function that we consider is defined 3 on $\{\nabla u \neq 0\}$ by

$$
\begin{aligned}
\mathscr{R}:= & -\frac{2 f(u) g(\nabla u, S u)|\nabla u|^{2}}{\Phi^{\prime}\left(|\nabla u|^{2}\right)}+2|\nabla u|^{2} \sum_{k=1}^{n} \sum_{j=1}^{N-n} g_{\eta_{j}}(\nabla u, S u) \frac{\partial S^{[j]} u}{\partial x_{k}} u_{k} \\
& +\frac{2 f(u)|\nabla u|^{2}}{\Lambda\left(|\nabla u|^{2}\right)} \sum_{j=1}^{n} g_{\zeta_{j}}(\nabla u, S u) u_{j} .
\end{aligned}
$$

As customary, if $N-n=0$ the second term in the right hand side of (1.14) is considered to be zero (equivalently, in this case, the function $g$ does not depend on the variable $\eta$ ).

Given $\alpha \in(0,1]$ we will also denote by $C^{0, \alpha}\left(\mathbb{R}^{n}\right)$ the space of functions $u \in L^{\infty}\left(\mathbb{R}^{n}\right)$ such that

$$
\sup _{\substack{x, y \in \mathbb{R}^{n} \\ x \neq y}} \frac{|u(x)-u(y)|}{|x-y|^{\alpha}}<+\infty \text {. }
$$

In this framework, our pivotal result is the following:

Theorem 1.2. Assume that $u \in C^{\ell}\left(\mathbb{R}^{n}\right) \cap W^{1, \infty}\left(\mathbb{R}^{n}\right)$ is a solution of (1.1). For every $r \in \mathbb{R}$, let

$$
F_{0}(r):=\int_{0}^{r} f(\tau) d \tau, \quad c_{u}:=\inf _{x \in \mathbb{R}^{n}} F_{0}(u(x)) \quad \text { and } \quad F(r):=F_{0}(r)-c_{u} .
$$

Assume that

$$
S u \in C^{0, \alpha}\left(\mathbb{R}^{n}, \mathbb{R}^{N-n}\right), \quad \text { for some } \alpha \in(0,1]
$$

and

$$
\mathscr{R}(x) \geq 0, \quad \text { for every } x \in\{\nabla u \neq 0\} \text {. }
$$

Then,

$$
2 \Phi^{\prime}\left(|\nabla u(x)|^{2}\right)|\nabla u(x)|^{2}-\Phi\left(|\nabla u(x)|^{2}\right) \leq 2 F(u(x)), \quad \text { for every } x \in \mathbb{R}^{n} .
$$

We observe that, since $u$ is bounded, we have that $c_{u}$ is finite and the setting in (1.15) is well posed. As a matter of fact, such a setting can be seen as a "gauge" on the potential function that makes $F$ nonnegative on the range of the solution.

Condition (1.16) can be seen as a regularity assumption on the solution (it can be also relaxed, for instance, if $S u(x)=(x, T u(x))$, with $T: L^{\infty}\left(\mathbb{R}^{n}\right) \cap C^{\ell}\left(\mathbb{R}^{n}\right) \mapsto\left(C^{\ell^{\prime}}\left(\mathbb{R}^{n}\right)\right)^{N-2 n}$, it is enough to suppose that $g$ is uniformly $C^{0, \alpha}$ in the $x$ variable and $\left.T u \in C^{0, \alpha}\left(\mathbb{R}^{n}, \mathbb{R}^{N-2 n}\right)\right)$.

We also point out that Theorem 1.2 comprises, as special cases, some classical results. In particular, when $\Phi(r):=r$ and $g$ vanishes identically, then $\mathscr{R}$ also vanishes identically, hence condition (1.17) is satisfied. In this case, equation (1.1) reduces to

$$
\Delta u=f(u)
$$

${ }^{3}$ We take this opportunity to amend a flaw in FV13. As a matter of fact, due to a cut-and-paste error, the term

$$
\frac{2 f(u)|\nabla u|^{2}}{\Lambda\left(|\nabla u|^{2}\right)} \sum_{j=1}^{n} g_{p_{j}}(x, u, \nabla u) u_{j}
$$

is missing from formula (1.13) in [FV13. The proof in FV13] (which is based on Lemma 2.1 there) is however correct as it is. Formula (1.11) and Remark 1.4 of [FV13] have also to be corrected by adding the missing term (e.g., saying that $f g_{p_{i}} p_{i} \geq 0$ ). Also, for clarity, we point out some minor typos in [FV13]: the statement " $w \in \mathcal{S}$ " three lines below (3.4) should be " $w \in \overline{\mathcal{S}}$ ", the set $V$ on line 3 of page 625 should be corrected into $\mathscr{V}$, the "neighborhood of $x_{0}$ " in the last line of the proof of Theorem 1.3 should be the "neighborhood of $y$ ". Also, throughout all [FV13, the function $g$ is assumed to be uniformly in $C^{0, \alpha}$ with respect to the $x$ variable. 
and (1.18) boils down to

$$
|\nabla u(x)|^{2} \leq 2 F(u(x)),
$$

which is precisely the classical result in [Mod85. Similarly, some results in [CGS94] and [FV13] are also recovered as particular cases of Theorem 1.2 (and, from the technical point of view, the setting introduced here simplifies and extends that in [FV13], by keeping track at the same time of all the derivatives of the nonlinear source $g$ ). In particular, recovering the elliptic regularity theory as mentioned in the footnote on page 3, one can obtain from Theorem 1.2 the classical results in [Mod85], [CGS94] and [FV13] also for weak solutions.

In some sense, one can consider Theorem 1.2 as an abstract result, in which a very general framework is taken into account, with minimal structural assumptions on the equation, but under a fundamental condition on the sign of the reminder function, as given in (1.17). To apply this result to particular cases of interest, we point out now that condition (1.17) is indeed satisfied in a number of concrete situations, such as the $p$-Laplacian operator, the graphical mean curvature operators, and operators obtained by the superposition of singular and degenerate operators with different scaling properties, proving gradient bounds under simple structural assumptions on the nonlinear sources. Indeed, we have the following result:

Proposition 1.3. Let $m \geq 1$ and $\Phi$ be as in (1.8), under assumptions (1.9) and (1.10), and suppose that $b_{k} \geq 0$ for all $k \in\{1, \ldots, m\}$. Assume that

$$
S(u):=u
$$

Suppose also that

$$
\begin{aligned}
& g(\zeta, \eta) \leq g(\zeta, \tilde{\eta}) \text { for all } \zeta \in \mathbb{R}^{n} \text { and } \eta \leq \tilde{\eta} \\
& \text { and } g(\lambda \zeta, \eta)=\lambda^{\beta} g(\zeta, \eta) \text { for all } \lambda>0, \text { for some } \beta>0
\end{aligned}
$$

In addition, assume that one of the following five conditions is satisfied: either

$$
m=1, \beta=p_{1}-1 \text { and }\left(p_{1}-2\right) f(\eta) g(\zeta, \eta) \geq 0, \text { for all } \zeta \in \mathbb{R}^{n} \text { and } \eta \in \mathbb{R},
$$

or

$$
m=1, p_{1}=2 \text { and }(\beta-1) f(\eta) g(\zeta, \eta) \geq 0 \text {, for all } \zeta \in \mathbb{R}^{n} \text { and } \eta \in \mathbb{R},
$$

or

$$
m=1, \beta=1 \text { and }\left(2-p_{1}\right) f(\eta) g(\zeta, \eta) \geq 0 \text {, for all } \zeta \in \mathbb{R}^{n} \text { and } \eta \in \mathbb{R},
$$

or

$$
\beta \geq \max \left\{1, p_{m}-1\right\} \text { and } f(\eta) g(\zeta, \eta) \geq 0, \text { for all } \zeta \in \mathbb{R}^{n} \text { and } \eta \in \mathbb{R}
$$

or

$$
b_{1}=\cdots=b_{m}=0, \beta \leq p_{1}-1 \text { and } f(\eta) g(\zeta, \eta) \leq 0 \text {, for all } \zeta \in \mathbb{R}^{n} \text { and } \eta \in \mathbb{R}
$$

or

$$
m=1, b_{1}=0 \text { and } \beta=p_{1}-1
$$

Then $\mathscr{R} \geq 0$.

A concrete example that satisfies assumptions (1.20) and (1.21) is

$$
g(\zeta, \eta)=|\zeta|^{\beta} h(\eta)
$$


with $\beta>1$ and $h$ increasing.

Other concrete situations in which one can explicitly check that $\mathscr{R} \geq 0$ will be discussed in the forthcoming Remarks 1.5 and 1.6.

Combining Theorem 1.2 with Propositions 1.1 and 1.3, we plainly obtain the following gradient estimate in a very general, but concrete, situation:

Corollary 1.4. Let $m \geq 1$ and

$$
\Phi(r):=\sum_{k=1}^{m}\left(\frac{2 c_{k}}{p_{k}}\left(b_{k}+r\right)^{\frac{p_{k}}{2}}-\frac{2 c_{k} b_{k}^{\frac{p_{k}}{2}}}{p_{k}}\right),
$$

with $1 \leq p_{1} \leq \ldots \leq p_{m}<+\infty$ and $c_{k}>0$ for every $k \in\{1, \ldots, m\}$.

Suppose that either

$$
\begin{array}{ll} 
& p_{1}>1, \quad \\
\text { and } \quad \mu b_{1} \leq b_{k} \leq \frac{b_{1}}{\mu}, \quad \text { for all } k \in\{1, \ldots, m\}
\end{array}
$$

or

$$
\mu \leq b_{k} \leq \frac{1}{\mu}, \quad \text { for all } k \in\{1, \ldots, m\}
$$

for some $\mu \in(0,1)$.

Suppose also that $g: \mathbb{R}^{n} \times \mathbb{R} \rightarrow \mathbb{R}$ satisfies the following monotonicity and homogeneity assumptions:

$$
\begin{aligned}
& g(\zeta, \eta) \leq g(\zeta, \tilde{\eta}) \text { for all } \zeta \in \mathbb{R}^{n} \text { and } \eta \leq \tilde{\eta}, \\
& \text { and } g(\lambda \zeta, \eta)=\lambda^{\beta} g(\zeta, \eta) \text { for all } \lambda, \beta>0
\end{aligned}
$$

In addition, assume that one of the following five conditions is satisfied: either

$$
m=1, \beta=p_{1}-1 \text { and }\left(p_{1}-2\right) f(\eta) g(\zeta, \eta) \geq 0, \text { for all } \zeta \in \mathbb{R}^{n} \text { and } \eta \in \mathbb{R},
$$

or

$$
m=1, p_{1}=2 \text { and }(\beta-1) f(\eta) g(\zeta, \eta) \geq 0 \text {, for all } \zeta \in \mathbb{R}^{n} \text { and } \eta \in \mathbb{R},
$$

or

$$
\begin{gathered}
m=1, \beta=1 \text { and }\left(2-p_{1}\right) f(\eta) g(\zeta, \eta) \geq 0, \text { for all } \zeta \in \mathbb{R}^{n} \text { and } \eta \in \mathbb{R}, \\
\beta \geq \max \left\{1, p_{m}-1\right\} \text { and } f(\eta) g(\zeta, \eta) \geq 0, \text { for all } \zeta \in \mathbb{R}^{n} \text { and } \eta \in \mathbb{R},
\end{gathered}
$$

or

$$
b_{1}=\cdots=b_{m}=0, \beta \leq p_{1}-1 \text { and } f(\eta) g(\zeta, \eta) \leq 0, \text { for all } \zeta \in \mathbb{R}^{n} \text { and } \eta \in \mathbb{R}
$$

or

$$
m=1, b_{1}=0 \text { and } \beta=p_{1}-1 .
$$

Assume that $u \in C^{\ell}\left(\mathbb{R}^{n}\right) \cap W^{1, \infty}\left(\mathbb{R}^{n}\right)$ is a solution of

$$
\operatorname{div}\left(\Phi^{\prime}\left(|\nabla u|^{2}\right) \nabla u\right)=f(u)+g(\nabla u, u), \quad \text { in } \mathbb{R}^{n} .
$$

For every $r \in \mathbb{R}$, let

$$
F_{0}(r):=\int_{0}^{r} f(\tau) d \tau, \quad c_{u}:=\inf _{x \in \mathbb{R}^{n}} F_{0}(u(x)) \quad \text { and } \quad F(r):=F_{0}(r)-c_{u} .
$$

Then,

$$
2 \Phi^{\prime}\left(|\nabla u(x)|^{2}\right)|\nabla u(x)|^{2}-\Phi\left(|\nabla u(x)|^{2}\right) \leq 2 F(u(x)), \quad \text { for every } x \in \mathbb{R}^{n}
$$


Remark 1.5. Checking condition (1.17) can be, in principle, not a trivial task in practice. Nevertheless, there are a number of concrete cases in which condition (1.17) is automatically satisfied. Without any attempt of being exhaustive, and only for the sake of confirming the interest of such a condition, we list here some of these situations in which condition (1.17) is fulfilled. For simplicity, we focus here on the case in which $f$ vanishes identically, and thus (1.14) reduces to

$$
\frac{\mathscr{R}}{2|\nabla u|^{2}}=\sum_{k=1}^{n} \sum_{j=1}^{N-n} g_{\eta_{j}}(\nabla u, S u) \frac{\partial S^{[j]} u}{\partial x_{k}} u_{k}
$$

(i). An interesting example is given by the equation

$$
\operatorname{div}\left(\Phi^{\prime}\left(|\nabla u|^{2}\right) \nabla u\right)=h(u)+c(x) \cdot \nabla u \quad \text { in } \mathbb{R}^{n},
$$

with $h \in C^{1}(\mathbb{R}), h^{\prime} \geq 0, c=\left(c_{1}, \ldots, c_{n}\right) \in C^{1}\left(\mathbb{R}^{n}, \mathbb{R}^{n}\right) \cap L^{\infty}\left(\mathbb{R}^{n}, \mathbb{R}^{n}\right)$, under the assumption that the matrix $\left\{\frac{\partial c_{j}}{\partial x_{k}}\right\}_{j, k \in\{1, \ldots, n\}}$ is nonnegative definite.

To check that (1.17) is satisfied in this case, it is convenient to take $N:=2 n+1, S u:=(c, h(u))$, that is

$$
S^{[j]} u:= \begin{cases}c_{j} & \text { if } j \in\{1, \ldots, n\} \\ h(u) & \text { if } j=n+1\end{cases}
$$

and

$$
g(\zeta, \eta)=g\left(\zeta_{1}, \ldots, \zeta_{n}, \eta_{1}, \ldots, \eta_{n+1}\right):=\sum_{j=1}^{n} \zeta_{j} \eta_{j}+\eta_{n+1} .
$$

Notice that, with this choice, the general setting in (1.1) gives precisely (1.41).

To check that condition (1.17) is satisfied in this case, we point out that for every $j \in\{1, \ldots, n\}$ we have that $g_{\eta_{j}}(\zeta, \eta)=\zeta_{j}$, and $g_{\eta_{n+1}}(\zeta, \eta)=1$. Accordingly,

$$
g_{\eta_{j}}(\nabla u, S u)= \begin{cases}u_{j} & \text { if } j \in\{1, \ldots, n\} \\ 1 & \text { if } j=n+1\end{cases}
$$

Furthermore,

$$
\frac{\partial S^{[j]} u}{\partial x_{k}}= \begin{cases}\frac{\partial c_{j}}{\partial x_{k}} & \text { if } j \in\{1, \ldots, n\} \\ h^{\prime}(u) u_{k} & \text { if } j=n+1 .\end{cases}
$$

Consequently, by (1.40),

$$
\begin{aligned}
\frac{\mathscr{R}}{2|\nabla u|^{2}} & =\sum_{k=1}^{n} \sum_{j=1}^{n+1} g_{\eta_{j}}(\nabla u, S u) \frac{\partial S^{[j]} u}{\partial x_{k}} u_{k} \\
& =\sum_{k=1}^{n}\left[\sum_{j=1}^{n} g_{\eta_{j}}(\nabla u, S u) \frac{\partial S^{[j]} u}{\partial x_{k}} u_{k}+g_{\eta_{n+1}}(\nabla u, S u) \frac{\partial S^{[n+1]} u}{\partial x_{k}} u_{k}\right] \\
& =\sum_{k=1}^{n}\left[\sum_{j=1}^{n} \frac{\partial c_{j}}{\partial x_{k}} u_{j} u_{k}+h^{\prime}(u) u_{k}^{2}\right] \\
& \geq 0 .
\end{aligned}
$$


This generalizes the result in (1.10) of [FV13] to more general operators.

(ii). As a further example, one can assume that

$$
g=g(\zeta, \eta) \text { is nondecreasing in } \eta
$$

and consider the projection operator

$$
S u(x):=u\left(x_{1}, 0, \ldots, 0\right) .
$$

In this case, condition (1.17) is satisfied by all solutions which are nondecreasing in the first direction, since, by (1.40),

$$
\frac{\mathscr{R}}{2|\nabla u|^{2}}=g_{\eta}(\nabla u, S u) \frac{\partial S u}{\partial x_{1}} u_{1}=g_{\eta}(\nabla u, S u) v_{1} u_{1} \geq 0,
$$

with $v_{1}(x):=u_{1}\left(x_{1}, 0, \ldots, 0\right)$.

(iii). Another interesting case is when (1.42) holds true and one considers the integral operator

$$
S u(x):=\int_{0}^{x_{1}} u\left(t, x_{2}, \ldots, x_{n}\right) d t
$$

and then (1.17) is satisfied by all nonnegative solutions which are nondecreasing in every direction (i.e., $u_{i} \geq 0$ for all $i \in\{1, \ldots, n\}$ ).

Indeed, in this case we have that

$$
v_{k}(x):=\int_{0}^{x_{1}} u_{k}\left(t, x_{2}, \ldots, x_{n}\right) d t \geq 0, \quad k=2, \ldots, n
$$

and hence, by (1.40),

$$
\begin{aligned}
\frac{\mathscr{R}}{2|\nabla u|^{2}} & =\sum_{k=1}^{n} g_{\eta}(\nabla u, S u) \frac{\partial S u}{\partial x_{k}} u_{k} \\
& =g_{\eta}(\nabla u, S u)\left(u u_{1}+\sum_{k=2}^{n} v_{k} u_{k}\right) \\
& \geq 0 .
\end{aligned}
$$

(iv). One can also assume (1.42) and take into account the convolution operator

$$
S u(x):=\int_{\mathbb{R}^{n}} u(x-y) K(y) d y,
$$

with $K \in C_{0}^{\infty}\left(\mathbb{R}^{n},[0,+\infty)\right)$. In this case, condition (1.17) is satisfied by all solutions which are nondecreasing in every direction, since

$$
v_{k}(x):=\int_{\mathbb{R}^{n}} u_{k}(x-y) K(y) d y \geq 0,
$$

and (1.40) gives that

$$
\frac{\mathscr{R}}{2|\nabla u|^{2}}=\sum_{k=1}^{n} g_{\eta}(\nabla u, S u) \frac{\partial S u}{\partial x_{k}} u_{k}=g_{\eta}(\nabla u, S u) \sum_{k=1}^{n} v_{k} u_{k} \geq 0
$$


(v). More generally, one can also assume (1.42) and take into account the multi-convolution operator

$$
S u(x):=\int_{\left(\mathbb{R}^{n}\right)^{d}} u\left(x-y_{1}\right) \ldots u\left(x-y_{d}\right) K\left(y_{1}, \ldots, y_{d}\right) d y_{1} \ldots d y_{d},
$$

with $K \in C_{0}^{\infty}\left(\left(\mathbb{R}^{n}\right)^{d},[0,+\infty)\right)$. Then, condition (1.17) is satisfied by all solutions which are nonnegative, and nondecreasing in every direction, since

$$
\begin{aligned}
v_{k}(x) & :=\partial_{x_{k}} \int_{\left(\mathbb{R}^{n}\right)^{d}} u\left(x-y_{1}\right) \ldots u\left(x-y_{d}\right) K\left(y_{1}, \ldots, y_{d}\right) d y_{1} \ldots d y_{d} \\
& =\sum_{h=1}^{d} \int_{\left(\mathbb{R}^{n}\right)^{d}} u_{k}\left(x-y_{h}\right)\left(\prod_{\substack{1 \leq j \leq d \\
j \neq h}} u\left(x-y_{j}\right)\right) K\left(y_{1}, \ldots, y_{d}\right) d y_{1} \ldots d y_{d} \\
& \geq 0
\end{aligned}
$$

and hence (1.40) gives that

$$
\frac{\mathscr{R}}{2|\nabla u|^{2}}=\sum_{k=1}^{n} g_{\eta}(\nabla u, S u) \frac{\partial S u}{\partial x_{k}} u_{k}=g_{\eta}(\nabla u, S u) \sum_{k=1}^{n} v_{k} u_{k} \geq 0 .
$$

(vi). Another interesting example is given by the equation

$$
\operatorname{div}\left(\Phi^{\prime}\left(|\nabla u|^{2}\right) \nabla u\right)=g\left(\nabla u,|u|^{q-1} u\right) \quad \text { in } \mathbb{R}^{n},
$$

with $q \geq 1$ and $g=g\left(\zeta_{1}, \ldots, \zeta_{n}, \eta\right)$ such that $g_{\eta} \geq 0$.

In this case, one takes $N:=n+1$ and $S u:=|u|^{q-1} u$. Then

$$
\frac{\partial S u}{\partial x_{k}}=q|u|^{q-1} u_{k}
$$

and hence, by (1.40),

$$
\frac{\mathscr{R}}{2|\nabla u|^{2}}=\sum_{k=1}^{n} g_{\eta}(\nabla u, S u) \frac{\partial S u}{\partial x_{k}} u_{k}=q g_{\eta}(\nabla u, S u)|u|^{q-1}|\nabla u|^{2} \geq 0 .
$$

Remark 1.6. An interesting example satisfying the structural assumption in (1.17) is provided by the equation

$$
\Delta u=f(u)+(c \cdot \nabla u) h(u),
$$

with $c \in \mathbb{R}^{n}, f, h \in C^{1}\left(\mathbb{R}^{n}\right)$ and $h^{\prime} \geq 0$. In this case, assumption (1.17) is fulfilled if $u$ is monotone nondecreasing in direction $c$, i.e. $c \cdot \nabla u \geq 0$.

Indeed, in this case we can take $\Phi(r):=r, N:=n+1, g(\zeta, \eta)=g\left(\zeta_{1}, \ldots, \zeta_{n}, \eta\right):=(c \cdot \zeta) \eta$ and $S u:=h(u)$. Then, the general equation in (1.1) reduces in this setting to the one in (1.44).

We observe that $g_{\zeta_{j}}(\zeta, \eta)=c_{j} \eta$ for all $j \in\{1, \ldots, n\}$ and $g_{\eta}(\zeta, \eta)=c \cdot \zeta$. Moreover, by (1.13), we see that $\Lambda(r)=1$. Consequently, we deduce from (1.14) that

$$
\begin{aligned}
\mathscr{R} & =-2 f(u) g(\nabla u, S u)|\nabla u|^{2}+2|\nabla u|^{2} \sum_{k=1}^{n} g_{\eta}(\nabla u, S u) \frac{\partial S u}{\partial x_{k}} u_{k}+2 f(u)|\nabla u|^{2} \sum_{j=1}^{n} g_{\zeta_{j}}(\nabla u, S u) u_{j} \\
& =-2 f(u)(c \cdot \nabla u) h(u)|\nabla u|^{2}+2|\nabla u|^{2} \sum_{k=1}^{n}(c \cdot \nabla u) h^{\prime}(u) u_{k}^{2}+2 f(u)|\nabla u|^{2} \sum_{j=1}^{n} c_{j} u_{j} h(u)
\end{aligned}
$$




$$
\begin{aligned}
& =2|\nabla u|^{2} \sum_{k=1}^{n}(c \cdot \nabla u) h^{\prime}(u) u_{k}^{2} \\
& \geq 0,
\end{aligned}
$$

and thus condition (1.17) is satisfied in this case as well.

Following some classical lines of research in [Mod85, CGS94,FV13] one has that pointwise gradient bounds are often related to classification results, since attaining the potential gauge at some point provides a very rigid information that can completely determine the solution. This is the counterpart of the fact that particles subject to ordinary differential equations remain motionless if they start with zero velocity at a potential well. In our setting, the corresponding result in this direction goes as follows:

Theorem 1.7. Let $u \in W^{1, \infty}\left(\mathbb{R}^{n}\right)$ and let the setting in (1.15) hold true. Assume also that (1.18) is satisfied.

Let $x_{0} \in \mathbb{R}^{n}$ be such that $u\left(x_{0}\right)=r_{0}$, with $F\left(r_{0}\right)=0$ and $F^{\prime}\left(r_{0}\right)=0$.

If $p>2$ in Assumption A, suppose also that

$$
\limsup _{r \rightarrow r_{0}} \frac{\left|F^{\prime}(r)\right|}{\left|r-r_{0}\right|^{p-1}}<+\infty .
$$

Then $u$ is constantly equal to $r_{0}$.

We observe that condition (1.45) cannot be dropped: indeed, if $p>2$ and

$$
\beta>\max \left\{2, \frac{p}{p-2}\right\}
$$

the function

$$
u: \mathbb{R}^{n} \mapsto \mathbb{R}, u(x)=|x|^{\beta}
$$

satisfies

$$
\begin{aligned}
& \operatorname{div}\left(|\nabla u|^{p-2} \nabla u\right)=\operatorname{div}\left(\left(\beta|x|^{\beta-1}\right)^{p-2}|x|^{\beta-2} \beta x\right)=\beta^{p-1} \operatorname{div}\left(|x|^{\beta p-\beta-p} x\right) \\
& \quad=\beta^{p-1}(\beta p-\beta-p+n)|x|^{\beta p-\beta-p}=\beta^{p-1}(\beta p-\beta-p+n)|u|^{\frac{\beta p-2 \beta-p}{\beta}} u=F^{\prime}(u),
\end{aligned}
$$

with

$$
F(r):=\frac{\beta^{p}(\beta p-\beta-p+n)}{(\beta-1) p}|r|^{\frac{(\beta-1) p}{\beta}} .
$$

Notice that in this case $F(u(0))=F(0)=0$, and $F^{\prime}(0)=0$, but $u$ is not constant, and (1.45) is violated since

$$
\lim _{r \rightarrow 0} \frac{F^{\prime}(r)}{|r|^{p-1}}=\lim _{r \rightarrow 0} \frac{\beta^{p}(\beta p-\beta-p+n)}{\beta}|r|^{-\frac{p}{\beta}}=+\infty .
$$

The rest of the paper is organized as follows. In Section 2, we show that Assumptions A and B are satisfied in several cases of interest, by proving Proposition 1.1.

Section 3 introduces the notion of $P$-function relative to equation (1.1) and contains the computations needed to check that such a function satisfies a suitable differential inequality, possibly in terms of the remainder $\mathscr{R}$.

Then, the proof of Theorem 1.2 is presented in Section 4 , while Section 5 is devoted to the proofs of Proposition 1.3 and Corollary 1.4, and Section 6 contains the proof of Theorem 1.7. 


\section{Structural assumptions and proof of Proposition 1.1}

In this section, we will establish Proposition [1.1. This will be accomplished in Propositions 2.1 and 2.2 (which will give, under suitable structural conditions, the setting in Assumption A), and in Propositions 2.3 and 2.4 (which will give, under suitable structural conditions, the setting in Assumption B). The precise computational details go as follows.

Proposition 2.1. Assume (1.9) and (1.10) hold true. Suppose also that

$$
\begin{gathered}
p_{1}>1, \\
b_{1} \geq 0 \quad \text { and } \quad \mu b_{1} \leq b_{k} \leq \frac{b_{1}}{\mu}, \quad \text { for all } k \in\{1, \ldots, m\},
\end{gathered}
$$

for some $\mu \in(0,1)$.

Let also

$$
\sigma \in B_{M} \backslash\{0\}
$$

for some $M \geq 1$. Then we have that

$$
C_{1}\left(\sqrt{b_{1}}+|\sigma|\right)^{p_{1}-2} \leq \Phi^{\prime}\left(|\sigma|^{2}\right) \leq C_{2}\left(\sqrt{b_{1}}+|\sigma|\right)^{p_{1}-2},
$$

where

$$
\begin{aligned}
C_{1} & :=c_{1}\left(\frac{1}{2}\right)^{\frac{\left|p_{1}-2\right|}{2}} \in(0,+\infty) \\
\text { and } \quad & C_{2}:=\left(\frac{2}{\mu}\right)^{\frac{\left|p_{1}-2\right|}{2}} \sum_{k=1}^{m} c_{k}\left(\frac{b_{1}}{\mu}+M^{2}\right)^{\frac{p_{k}-p_{1}}{2}} \in(0,+\infty) .
\end{aligned}
$$

Proof. By (1.8), we have

$$
\Phi^{\prime}(r)=\sum_{k=1}^{m} c_{k}\left(b_{k}+r\right)^{\frac{p_{k}-2}{2}} .
$$

As a consequence,

$$
\Phi^{\prime}\left(|\sigma|^{2}\right)=\sum_{k=1}^{m} c_{k}\left(b_{k}+|\sigma|^{2}\right)^{\frac{p_{k}-2}{2}} .
$$

In addition, we observe

$$
q_{1}^{2}+q_{2}^{2} \leq\left(q_{1}+q_{2}\right)^{2} \leq 2\left(q_{1}^{2}+q_{2}^{2}\right) \text { for all } q_{1}, q_{2} \in[0,+\infty),
$$

therefore

$$
\beta_{0}+|\sigma|^{2} \leq\left(\sqrt{\beta_{0}}+|\sigma|\right)^{2} \leq 2\left(\beta_{0}+|\sigma|^{2}\right),
$$

for all $\beta_{0} \geq 0$.

Now, to establish the upper bound in (2.4), we use (2.8) and observe that

$$
\Phi^{\prime}\left(|\sigma|^{2}\right) \leq \sum_{k=1}^{m} c_{k}\left(b_{k}+|\sigma|^{2}\right)^{\frac{p_{1}-2}{2}}\left(\frac{b_{1}}{\mu}+M^{2}\right)^{\frac{p_{k}-p_{1}}{2}} .
$$


Now we claim

$$
\left(b_{k}+|\sigma|^{2}\right)^{\frac{p_{1}-2}{2}} \leq \frac{1}{\mu^{\frac{\left|p_{1}-2\right|}{2}}}\left(b_{1}+|\sigma|^{2}\right)^{\frac{p_{1}-2}{2}} .
$$

Indeed, if $p_{1} \geq 2$, we recall (2.2) and have that

$$
b_{k}+|\sigma|^{2} \leq \frac{b_{1}}{\mu}+|\sigma|^{2} \leq \frac{1}{\mu}\left(b_{1}+|\sigma|^{2}\right),
$$

which gives (2.11). If instead $p_{1}<2$, we use the inequality

$$
b_{k}+|\sigma|^{2} \geq \mu b_{1}+|\sigma|^{2} \geq \mu\left(b_{1}+|\sigma|^{2}\right),
$$

and this gives (2.11) in this case as well.

Then, we insert (2.11) into (2.10) and find that

$$
\Phi^{\prime}\left(|\sigma|^{2}\right) \leq \frac{\left(b_{1}+|\sigma|^{2}\right)^{\frac{p_{1}-2}{2}}}{\mu^{\frac{\left|p_{1}-2\right|}{2}}} \sum_{k=1}^{m} c_{k}\left(\frac{b_{1}}{\mu}+M^{2}\right)^{\frac{p_{k}-p_{1}}{2}} .
$$

This and (2.9) give

$$
\Phi^{\prime}\left(|\sigma|^{2}\right) \leq\left(\frac{2}{\mu}\right)^{\frac{\left|p_{1}-2\right|}{2}}\left(\sqrt{b_{1}}+|\sigma|\right)^{p_{1}-2} \sum_{k=1}^{m} c_{k}\left(\frac{b_{1}}{\mu}+M^{2}\right)^{\frac{p_{k}-p_{1}}{2}} .
$$

From this and (2.6) we conclude that the upper bound in (2.4) is satisfied, as desired.

Now we check the lower bound in (2.4). For this, by (2.8) and (2.9), we have

$$
\Phi^{\prime}\left(|\sigma|^{2}\right) \geq c_{1}\left(b_{1}+|\sigma|^{2}\right)^{\frac{p_{1}-2}{2}} \geq c_{1}\left(\frac{1}{2}\right)^{\frac{\left|p_{1}-2\right|}{2}}\left(\sqrt{b_{1}}+|\sigma|\right)^{p_{1}-2} .
$$

This and (2.5) give the lower bound in (2.4).

Proposition 2.2. Assume (1.9) and (1.10) hold true. Suppose also that (2.1), (2.2) and (2.3) are satisfied. Then, for every $\xi \in \mathbb{R}^{n}$ we have that

$$
C_{1}\left(\sqrt{b_{1}}+|\sigma|\right)^{p_{1}-2}|\xi|^{2} \leq \sum_{i, j=1}^{n} a_{i j}(\sigma) \xi_{i} \xi_{j} \leq C_{2}\left(\sqrt{b_{1}}+|\sigma|\right)^{p_{1}-2}|\xi|^{2},
$$

where

$$
\begin{aligned}
C_{1} & :=c_{1} \min \left\{1, p_{1}-1\right\}\left(\frac{\mu}{2}\right)^{\frac{\left|p_{1}-2\right|}{2}} \in(0,+\infty) \\
\text { and } \quad C_{2} & :=\left(\frac{2}{\mu}\right)^{\frac{\left|p_{1}-2\right|}{2}}\left(p_{m}+1\right) \sum_{k=1}^{m} c_{k}\left(\frac{b_{1}}{\mu}+M^{2}\right)^{\frac{p_{k}-p_{1}}{2}} \in(0,+\infty) .
\end{aligned}
$$

Proof. First of all, from (2.7), we obtain

$$
\Phi^{\prime \prime}(r)=\sum_{k=1}^{m} \frac{c_{k}\left(p_{k}-2\right)}{2}\left(b_{k}+r\right)^{\frac{p_{k}-4}{2}}
$$


Accordingly, we have that

$$
a_{i j}(\sigma)=\sum_{k=1}^{m}\left[c_{k}\left(p_{k}-2\right)\left(b_{k}+|\sigma|^{2}\right)^{\frac{p_{k}-4}{2}} \sigma_{i} \sigma_{j}+c_{k}\left(b_{k}+|\sigma|^{2}\right)^{\frac{p_{k}-2}{2}} \delta_{i j}\right],
$$

and therefore, for every $\xi=\left(\xi_{1}, \ldots, \xi_{n}\right) \in \mathbb{R}^{n}$,

$$
\sum_{i, j=1}^{n} a_{i j}(\sigma) \xi_{i} \xi_{j}=\sum_{k=1}^{m}\left[c_{k}\left(p_{k}-2\right)\left(b_{k}+|\sigma|^{2}\right)^{\frac{p_{k}-4}{2}}(\sigma \cdot \xi)^{2}+c_{k}\left(b_{k}+|\sigma|^{2}\right)^{\frac{p_{k}-2}{2}}|\xi|^{2}\right] .
$$

To prove the upper bound in (2.12) we argue as follows. We exploit (1.9) to see that

$$
\begin{aligned}
\sum_{i, j=1}^{n} a_{i j}(\sigma) \xi_{i} \xi_{j} & \leq \sum_{k=1}^{m}\left[c_{k} p_{k}\left(b_{k}+|\sigma|^{2}\right)^{\frac{p_{k}-4}{2}}|\sigma|^{2}|\xi|^{2}+c_{k}\left(b_{k}+|\sigma|^{2}\right)^{\frac{p_{k}-2}{2}}|\xi|^{2}\right] \\
& \leq \sum_{k=1}^{m}\left[c_{k} p_{k}\left(b_{k}+|\sigma|^{2}\right)^{\frac{p_{k}-2}{2}}|\xi|^{2}+c_{k}\left(b_{k}+|\sigma|^{2}\right)^{\frac{p_{k}-2}{2}}|\xi|^{2}\right] \\
& =\sum_{k=1}^{m} c_{k}\left(p_{k}+1\right)\left(b_{k}+|\sigma|^{2}\right)^{\frac{p_{k}-2}{2}}|\xi|^{2} \\
& \leq\left(p_{m}+1\right) \sum_{k=1}^{m} c_{k}\left(b_{k}+|\sigma|^{2}\right)^{\frac{p_{k}-2}{2}}|\xi|^{2} .
\end{aligned}
$$

Furthermore, in view of (1.9),$(2.3)$ and (2.11), we see that

$$
\left(b_{k}+|\sigma|^{2}\right)^{\frac{p_{k}-2}{2}}=\left(b_{k}+|\sigma|^{2}\right)^{\frac{p_{1}-2}{2}}\left(b_{k}+|\sigma|^{2}\right)^{\frac{p_{k}-p_{1}}{2}} \leq \frac{1}{\mu^{\frac{\left|p_{1}-2\right|}{2}}}\left(b_{1}+|\sigma|^{2}\right)^{\frac{p_{1}-2}{2}}\left(\frac{b_{1}}{\mu}+M^{2}\right)^{\frac{p_{k}-p_{1}}{2}} .
$$

Consequently, by (2.9), we have

$$
\left(b_{k}+|\sigma|^{2}\right)^{\frac{p_{k}-2}{2}} \leq\left(\frac{2}{\mu}\right)^{\frac{\left|p_{1}-2\right|}{2}}\left(\sqrt{b_{1}}+|\sigma|\right)^{p_{1}-2}\left(\frac{b_{1}}{\mu}+M^{2}\right)^{\frac{p_{k}-p_{1}}{2}} .
$$

Hence (2.17) gives that

$$
\sum_{i, j=1}^{n} a_{i j}(\sigma) \xi_{i} \xi_{j} \leq\left(\frac{2}{\mu}\right)^{\frac{\left|p_{1}-2\right|}{2}}\left(p_{m}+1\right)|\xi|^{2}\left(\sqrt{b_{1}}+|\sigma|\right)^{p_{1}-2} \sum_{k=1}^{m} c_{k}\left(\frac{b_{1}}{\mu}+M^{2}\right)^{\frac{p_{k}-p_{1}}{2}} .
$$

This together with (2.14) establishes the upper bound in (2.12), and we now deal with the lower bound in (2.12). To this end, we observe that if $p_{k} \leq 2$, then

$$
\begin{aligned}
& c_{k}\left(p_{k}-2\right)\left(b_{k}+|\sigma|^{2}\right)^{\frac{p_{k}-4}{2}}(\sigma \cdot \xi)^{2}+c_{k}\left(b_{k}+|\sigma|^{2}\right)^{\frac{p_{k}-2}{2}}|\xi|^{2} \\
= & -c_{k}\left(2-p_{k}\right)\left(b_{k}+|\sigma|^{2}\right)^{\frac{p_{k}-4}{2}}(\sigma \cdot \xi)^{2}+c_{k}\left(b_{k}+|\sigma|^{2}\right)^{\frac{p_{k}-2}{2}}|\xi|^{2} \\
\geq & -c_{k}\left(2-p_{k}\right)\left(b_{k}+|\sigma|^{2}\right)^{\frac{p_{k}-4}{2}}|\sigma|^{2}|\xi|^{2}+c_{k}\left(b_{k}+|\sigma|^{2}\right)^{\frac{p_{k}-2}{2}}|\xi|^{2} \\
\geq & -c_{k}\left(2-p_{k}\right)\left(b_{k}+|\sigma|^{2}\right)^{\frac{p_{k}-4}{2}}\left(b_{k}+|\sigma|^{2}\right)|\xi|^{2}+c_{k}\left(b_{k}+|\sigma|^{2}\right)^{\frac{p_{k}-2}{2}}|\xi|^{2} \\
= & c_{k}\left(p_{k}-1\right)\left(b_{k}+|\sigma|^{2}\right)^{\frac{p_{k}-2}{2}}|\xi|^{2}
\end{aligned}
$$




$$
\begin{aligned}
& \geq c_{k}\left(p_{1}-1\right)\left(\frac{b_{1}}{\mu}+|\sigma|^{2}\right)^{\frac{p_{k}-2}{2}}|\xi|^{2} \\
& \geq c_{k} \mu^{\frac{2-p_{k}}{2}}\left(p_{1}-1\right)\left(b_{1}+|\sigma|^{2}\right)^{\frac{p_{k}-2}{2}}|\xi|^{2},
\end{aligned}
$$

thanks to (2.2).

This and (2.16) yield that

$$
\begin{aligned}
\sum_{i, j=1}^{n} a_{i j}(\sigma) \xi_{i} \xi_{j} \geq & \left(p_{1}-1\right) \sum_{\substack{1 \leq k \leq m \\
p_{k} \leq 2}} c_{k} \mu^{\frac{2-p_{k}}{2}}\left(b_{1}+|\sigma|^{2}\right)^{\frac{p_{k}-2}{2}}|\xi|^{2} \\
& \quad+\sum_{\substack{1 \leq k \leq m \\
p_{k}>2}}\left[c_{k}\left(p_{k}-2\right)\left(b_{k}+|\sigma|^{2}\right)^{\frac{p_{k}-4}{2}}(\sigma \cdot \xi)^{2}+c_{k}\left(b_{k}+|\sigma|^{2}\right)^{\frac{p_{k}-2}{2}}|\xi|^{2}\right] \\
\geq & \left(p_{1}-1\right) \sum_{\substack{1 \leq k \leq m \\
p_{k} \leq 2}} c_{k} \mu^{\frac{\left|p_{k}-2\right|}{2}}\left(b_{1}+|\sigma|^{2}\right)^{\frac{p_{k}-2}{2}}|\xi|^{2}+\sum_{1 \leq k \leq m} c_{k}\left(b_{k}+|\sigma|^{2}\right)^{\frac{p_{k}-2}{2}}|\xi|^{2} \\
\geq & \left(p_{1}-1\right) \sum_{\substack{1 \leq k \leq m \\
p_{k} \leq 2}} c_{k} \mu^{\frac{\left|p_{k}-2\right|}{2}}\left(b_{1}+|\sigma|^{2}\right)^{\frac{p_{k}-2}{2}}|\xi|^{2}+\sum_{\substack{1 \leq k \leq m \\
p_{k}>2}} c_{k}\left(\mu b_{1}+|\sigma|^{2}\right)^{\frac{p_{k}-2}{2}}|\xi|^{2} \\
\geq & \left(p_{1}-1\right) \sum_{\substack{1 \leq k \leq m \\
p_{k} \leq 2}} c_{k} \mu^{\frac{\left|p_{k}-2\right|}{2}}\left(b_{1}+|\sigma|^{2}\right)^{\frac{p_{k}-2}{2}}|\xi|^{2}+\sum_{\substack{1 \leq k \leq m \\
p_{k}>2}} c_{k} \mu^{\frac{p_{k}-2}{2}}\left(b_{1}+|\sigma|^{2}\right)^{\frac{p_{k}-2}{2}}|\xi|^{2} \\
= & \left(p_{1}-1\right) \sum_{\substack{1 \leq k \leq m \\
p_{k} \leq 2}} c_{k} \mu^{\frac{\left|p_{k}-2\right|}{2}}\left(b_{1}+|\sigma|^{2}\right)^{\frac{p_{k}-2}{2}}|\xi|^{2}+\sum_{\substack{1 \leq k \leq m \\
p_{k}>2}} c_{k} \mu^{\frac{\left|p_{k}-2\right|}{2}}\left(b_{1}+|\sigma|^{2}\right)^{\frac{p_{k}-2}{2}}|\xi|^{2} \\
\geq & \min \left\{1, p_{1}-1\right\} \sum_{k=1}^{m} c_{k} \mu^{\frac{\left|p_{k}-2\right|}{2}}\left(b_{1}+|\sigma|^{2}\right)^{\frac{p_{k}-2}{2}}|\xi|^{2} \\
\geq & \min \left\{1, p_{1}-1\right\} c_{1} \mu^{\frac{\left|p_{1}-2\right|}{2}}\left(b_{1}+|\sigma|^{2}\right)^{\frac{p_{1}-2}{2}}|\xi|^{2} .
\end{aligned}
$$

From this and (2.9) we obtain

$$
\sum_{i, j=1}^{n} a_{i j}(\sigma) \xi_{i} \xi_{j} \geq c_{1} \min \left\{1, p_{1}-1\right\}\left(\frac{\mu}{2}\right)^{\frac{\left|p_{1}-2\right|}{2}}\left(\sqrt{b_{1}}+|\sigma|\right)^{p_{1}-2}|\xi|^{2} .
$$

This gives the lower bound in (2.12), thanks to the setting in (2.13), and we stress that $C_{1}>0$, in light of $(\underline{2.1})$.

Proposition 2.3. Assume (1.9) and (1.10) hold true. Suppose also that

$$
\mu \leq b_{k} \leq \frac{1}{\mu}, \quad \text { for all } k \in\{1, \ldots, m\}
$$

for some $\mu \in(0,1)$.

Let also

$$
\sigma \in B_{M} \backslash\{0\}
$$

for some $M \geq 1$. Then we have that

$$
C_{1}(1+|\sigma|)^{-1} \leq \Phi^{\prime}\left(|\sigma|^{2}\right) \leq C_{2}(1+|\sigma|)^{-1}
$$


where

$$
\begin{aligned}
C_{1} & :=c_{1} \mu^{\frac{p_{1}}{2}} \in(0,+\infty) \\
\text { and } \quad C_{2} & :=\sqrt{\frac{2}{\mu}} \sum_{k=1}^{m} c_{k}\left(\frac{1}{\mu}+M^{2}\right)^{\frac{p_{k}-1}{2}} \in(0,+\infty) .
\end{aligned}
$$

Proof. We use (1.9), (2.18) and (2.19) to obtain that

$$
\begin{aligned}
& \left(b_{k}+|\sigma|^{2}\right)^{\frac{p_{k}-2}{2}}=\left(b_{k}+|\sigma|^{2}\right)^{-\frac{1}{2}}\left(b_{k}+|\sigma|^{2}\right)^{\frac{p_{k}-1}{2}} \leq\left(\mu+|\sigma|^{2}\right)^{-\frac{1}{2}}\left(\frac{1}{\mu}+M^{2}\right)^{\frac{p_{k}-1}{2}} \\
& \quad \leq \frac{1}{\sqrt{\mu}}\left(1+|\sigma|^{2}\right)^{-\frac{1}{2}}\left(\frac{1}{\mu}+M^{2}\right)^{\frac{p_{k}-1}{2}} .
\end{aligned}
$$

This and (2.9) yield

$$
\left(b_{k}+|\sigma|^{2}\right)^{\frac{p_{k}-2}{2}} \leq \sqrt{\frac{2}{\mu}}(1+|\sigma|)^{-1}\left(\frac{1}{\mu}+M^{2}\right)^{\frac{p_{k}-1}{2}} .
$$

Plugging this information into (2.8), we see that

$$
\Phi^{\prime}\left(|\sigma|^{2}\right) \leq \sqrt{\frac{2}{\mu}}(1+|\sigma|)^{-1} \sum_{k=1}^{m} c_{k}\left(\frac{1}{\mu}+M^{2}\right)^{\frac{p_{k}-1}{2}} .
$$

This and (2.22) give the upper bound in (2.20).

Furthermore, by (2.8) and (2.18), we have

$$
\begin{aligned}
\Phi^{\prime}\left(|\sigma|^{2}\right) & =\sum_{k=1}^{m} c_{k}\left(b_{k}+|\sigma|^{2}\right)^{\frac{p_{k}-2}{2}} \\
& \geq c_{1}\left(b_{1}+|\sigma|^{2}\right)^{\frac{p_{1}-2}{2}} \\
& =c_{1}\left(b_{1}+|\sigma|^{2}\right)^{-\frac{1}{2}}\left(b_{1}+|\sigma|^{2}\right)^{\frac{p_{1}-1}{2}} \\
& \geq c_{1}\left(\frac{1}{\mu}+|\sigma|^{2}\right)^{-\frac{1}{2}} \mu^{\frac{p_{1}-1}{2}} \\
& \geq c_{1} \sqrt{\mu}\left(1+|\sigma|^{2}\right)^{-\frac{1}{2}} \mu^{\frac{p_{1}-1}{2}} .
\end{aligned}
$$

This and (2.9) lead to

$$
\Phi^{\prime}\left(|\sigma|^{2}\right) \geq c_{1}(1+|\sigma|)^{-1} \mu^{\frac{p_{1}}{2}} .
$$

Hence, recalling (2.21), we obtain the lower bound in (2.20), as desired.

Proposition 2.4. Assume (1.9) and (1.10) hold true. Suppose also that (2.18) and (2.19) are satisfied. Then, for every $\xi^{\prime}=\left(\xi_{1}, \ldots, \xi_{n+1}\right)=\left(\xi, \xi_{n+1}\right) \in \mathbb{R}^{n} \times \mathbb{R}$ with $\xi \cdot \sigma=\xi_{n+1}$, we have that

$$
C_{1}(1+|\sigma|)^{-1}\left|\xi^{\prime}\right|^{2} \leq \sum_{i, j=1}^{n} a_{i j}(\sigma) \xi_{i} \xi_{j} \leq C_{2}(1+|\sigma|)^{-1}\left|\xi^{\prime}\right|^{2}
$$


where

$$
\begin{aligned}
C_{1} & :=\frac{c_{1} \min \left\{1, p_{1}-1\right\}}{1+M^{2}} \mu^{\frac{p_{1}}{2}} \in(0,+\infty) \\
\text { and } \quad C_{2} & :=\sqrt{\frac{2}{\mu}} \sum_{k=1}^{m} c_{k}\left(\left|p_{k}-2\right|+1\right)\left(\frac{1}{\mu}+M^{2}\right)^{\frac{p_{k}-1}{2}} \in(0,+\infty) .
\end{aligned}
$$

Proof. The argument is a careful modification of that used in the proof of Proposition [2.2, taking into special consideration the $(n+1)$ th component of the vector $\xi^{\prime}$.

To prove the upper bound in (2.23), we recall (2.16) and perform the following computation:

$$
\begin{aligned}
\sum_{i, j=1}^{n} a_{i j}(\sigma) \xi_{i} \xi_{j} & \leq \sum_{k=1}^{m}\left[c_{k}\left|p_{k}-2\right|\left(b_{k}+|\sigma|^{2}\right)^{\frac{p_{k}-4}{2}}(\sigma \cdot \xi)^{2}+c_{k}\left(b_{k}+|\sigma|^{2}\right)^{\frac{p_{k}-2}{2}}|\xi|^{2}\right] \\
& \leq \sum_{k=1}^{m} c_{k}\left(\left|p_{k}-2\right|+1\right)\left(b_{k}+|\sigma|^{2}\right)^{\frac{p_{k}-2}{2}}|\xi|^{2} \\
& \leq \sum_{k=1}^{m} c_{k}\left(\left|p_{k}-2\right|+1\right)\left(b_{k}+|\sigma|^{2}\right)^{-\frac{1}{2}}\left(\frac{1}{\mu}+M^{2}\right)^{\frac{p_{k}-1}{2}}|\xi|^{2} \\
& \leq \sum_{k=1}^{m} c_{k}\left(\left|p_{k}-2\right|+1\right)\left(\mu+|\sigma|^{2}\right)^{-\frac{1}{2}}\left(\frac{1}{\mu}+M^{2}\right)^{\frac{p_{k}-1}{2}}|\xi|^{2} \\
& \leq \frac{1}{\sqrt{\mu}} \sum_{k=1}^{m} c_{k}\left(\left|p_{k}-2\right|+1\right)\left(1+|\sigma|^{2}\right)^{-\frac{1}{2}}\left(\frac{1}{\mu}+M^{2}\right)^{\frac{p_{k}-1}{2}}|\xi|^{2}
\end{aligned}
$$

thanks to (2.18) and (2.19). Hence, recalling (2.9), we have

$$
\sum_{i, j=1}^{n} a_{i j}(\sigma) \xi_{i} \xi_{j} \leq \sqrt{\frac{2}{\mu}} \sum_{k=1}^{m} c_{k}\left(\left|p_{k}-2\right|+1\right)(1+|\sigma|)^{-1}\left(\frac{1}{\mu}+M^{2}\right)^{\frac{p_{k}-1}{2}}|\xi|^{2} .
$$

This proves the upper bound in (2.23), in light of (2.25) and the fact that $|\xi| \leq\left|\xi^{\prime}\right|$.

Now we prove the lower bound in (2.23). For this, we use (2.19) to see that

$$
\left|\xi^{\prime}\right|^{2}=|\xi|^{2}+|\xi \cdot \sigma|^{2} \leq\left(1+M^{2}\right)|\xi|^{2}
$$

Also, if $p_{k} \leq 2$, then

$$
\begin{aligned}
& c_{k}\left(p_{k}-2\right)\left(b_{k}+|\sigma|^{2}\right)^{\frac{p_{k}-4}{2}}(\sigma \cdot \xi)^{2}+c_{k}\left(b_{k}+|\sigma|^{2}\right)^{\frac{p_{k}-2}{2}}|\xi|^{2} \\
\geq & -c_{k}\left(2-p_{k}\right)\left(b_{k}+|\sigma|^{2}\right)^{\frac{p_{k}-4}{2}}|\sigma|^{2}|\xi|^{2}+c_{k}\left(b_{k}+|\sigma|^{2}\right)^{\frac{p_{k}-2}{2}}|\xi|^{2} \\
\geq & -c_{k}\left(2-p_{k}\right)\left(b_{k}+|\sigma|^{2}\right)^{\frac{p_{k}-2}{2}}|\xi|^{2}+c_{k}\left(b_{k}+|\sigma|^{2}\right)^{\frac{p_{k}-2}{2}}|\xi|^{2} \\
= & c_{k}\left(p_{k}-1\right)\left(b_{k}+|\sigma|^{2}\right)^{\frac{p_{k}-2}{2}}|\xi|^{2} .
\end{aligned}
$$

This and (2.16) give that

$$
\sum_{i, j=1}^{n} a_{i j}(\sigma) \xi_{i} \xi_{j} \geq \sum_{\substack{1 \leq k \leq m \\ p_{k} \leq 2}} c_{k}\left(p_{k}-1\right)\left(b_{k}+|\sigma|^{2}\right)^{\frac{p_{k}-2}{2}}|\xi|^{2}
$$




$$
\begin{aligned}
& +\sum_{\substack{1 \leq k \leq m \\
p_{k}>2}}\left[c_{k}\left(p_{k}-2\right)\left(b_{k}+|\sigma|^{2}\right)^{\frac{p_{k}-4}{2}}(\sigma \cdot \xi)^{2}+c_{k}\left(b_{k}+|\sigma|^{2}\right)^{\frac{p_{k}-2}{2}}|\xi|^{2}\right] \\
\geq & \sum_{\substack{1 \leq k \leq m \\
p_{k} \leq 2}} c_{k}\left(p_{1}-1\right)\left(b_{k}+|\sigma|^{2}\right)^{\frac{p_{k}-2}{2}}|\xi|^{2}+\sum_{\substack{1 \leq k \leq m \\
p_{k}>2}} c_{k}\left(b_{k}+|\sigma|^{2}\right)^{\frac{p_{k}-2}{2}}|\xi|^{2} \\
\geq & \min \left\{1, p_{1}-1\right\} \sum_{k=1}^{m} c_{k}\left(b_{k}+|\sigma|^{2}\right)^{\frac{p_{k}-2}{2}}|\xi|^{2} \\
\geq & c_{1} \min \left\{1, p_{1}-1\right\}\left(b_{1}+|\sigma|^{2}\right)^{\frac{p_{1}-2}{2}}|\xi|^{2} .
\end{aligned}
$$

Hence, in view of (2.26), we get

$$
\begin{aligned}
\sum_{i, j=1}^{n} a_{i j}(\sigma) \xi_{i} \xi_{j} & \geq \frac{c_{1} \min \left\{1, p_{1}-1\right\}}{1+M^{2}}\left(b_{1}+|\sigma|^{2}\right)^{\frac{p_{1}-2}{2}}\left|\xi^{\prime}\right|^{2} \\
& \geq \frac{c_{1} \min \left\{1, p_{1}-1\right\}}{1+M^{2}}\left(b_{1}+|\sigma|^{2}\right)^{-\frac{1}{2}} \mu^{\frac{p_{1}-1}{2}}\left|\xi^{\prime}\right|^{2} \\
& \geq \frac{c_{1} \min \left\{1, p_{1}-1\right\}}{1+M^{2}}\left(\frac{1}{\mu}+|\sigma|^{2}\right)^{-\frac{1}{2}} \mu^{\frac{p_{1}-1}{2}}\left|\xi^{\prime}\right|^{2} \\
& \geq \frac{c_{1} \min \left\{1, p_{1}-1\right\}}{1+M^{2}}\left(1+|\sigma|^{2}\right)^{-\frac{1}{2}} \mu^{\frac{p_{1}}{2}}\left|\xi^{\prime}\right|^{2} .
\end{aligned}
$$

This and (2.9) give that

$$
\sum_{i, j=1}^{n} a_{i j}(\sigma) \xi_{i} \xi_{j} \geq \frac{c_{1} \min \left\{1, p_{1}-1\right\}}{1+M^{2}}(1+|\sigma|)^{-1} \mu^{\frac{p_{1}}{2}}\left|\xi^{\prime}\right|^{2}
$$

that is the lower bound in (2.23), thanks to (2.24).

By means of the above conclusions, we are in the position of proving Propostion 1.1:

Proof. The claim in (i) of Proposition 1.1 directly follows from Propositions 2.1 and 2.2. Similarly, the claim in (ii) of Proposition 1.1 is a consequence of Propositions 2.3 and 2.4.

\section{$3 \quad P$-function computations}

The goal of this section is to introduce an appropriate $P$-function relative to equation (1.1) and establish a differential inequality for it (combining this with the Maximum Principle, we will obtain also the desired gradient bounds). To implement this strategy, for such a solution $u$, for all $x \in \mathbb{R}^{n}$ we define

$$
P(u ; x):=2 \Phi^{\prime}\left(|\nabla u(x)|^{2}\right)|\nabla u(x)|^{2}-\Phi\left(|\nabla u(x)|^{2}\right)-2 F(u(x)),
$$

and we prove the following result:

Lemma 3.1. Let $\Omega$ be an open subset of $\mathbb{R}^{n}$. Let $u$ be a solution of $(1.1)$ in $\Omega$, with $\nabla u \neq 0$ in $\Omega$, and

$$
\Lambda(r)>0 \text { for all } r>0 \text {. }
$$


Let

$$
d_{i j}(\sigma):=\frac{a_{i j}(\sigma)}{\Lambda\left(|\sigma|^{2}\right)}
$$

and

$$
B_{i}(x)=B_{i}(u ; x):=-2 \frac{f(u)}{\Lambda\left(|\nabla u|^{2}\right)}\left(1+\frac{|\nabla u|^{2} \Phi^{\prime \prime}\left(|\nabla u|^{2}\right)}{\Phi^{\prime}\left(|\nabla u|^{2}\right)}\right) \frac{\partial u}{\partial x_{i}}-\frac{|\nabla u|^{2}}{\Lambda\left(|\nabla u|^{2}\right)} g_{\zeta_{i}}(\nabla u, S u) .
$$

Then, we have that

$$
\sum_{i, j}|\nabla u|^{2} \frac{\partial}{\partial x_{j}}\left(d_{i j}(\nabla u) \frac{\partial P}{\partial x_{i}}\right)+\sum_{i} B_{i} \frac{\partial P}{\partial x_{i}} \geq \frac{|\nabla P|^{2}}{2 \Lambda\left(|\nabla u|^{2}\right)}+\mathscr{R}, \quad \text { in } \Omega .
$$

Proof. By (3.2), the map $r \mapsto 2 \Phi^{\prime}(r) r-\Phi(r)$ is invertible, and we denote by $\Psi$ its inverse. Notice that

$$
\Psi(P(u ; x)+2 F(u(x)))=|\nabla u(x)|^{2} .
$$

Moreover, by the definition of $\Psi$ and (1.13), we have

$$
1=\frac{\mathrm{d}}{\mathrm{d} r}\left(\Psi\left(2 \Phi^{\prime}(r) r-\Phi(r)\right)\right)=\Psi^{\prime}\left(2 \Phi^{\prime}(r) r-\Phi(r)\right) \Lambda(r),
$$

hence

$$
\Psi^{\prime}\left(2 \Phi^{\prime}\left(|\nabla u|^{2}\right)|\nabla u|^{2}-\Phi\left(|\nabla u|^{2}\right)\right)=\frac{1}{\Lambda\left(|\nabla u|^{2}\right)} .
$$

Now, differentiating (3.1) and recalling (1.13), we see that

$$
\begin{aligned}
\frac{\partial P}{\partial x_{i}} & =2\left(2 \Phi^{\prime \prime}\left(|\nabla u(x)|^{2}\right)|\nabla u(x)|^{2}+\Phi^{\prime}\left(|\nabla u(x)|^{2}\right)\right)|\nabla u(x)| \frac{\partial|\nabla u(x)|}{\partial x_{i}}-2 f(u) \frac{\partial u}{\partial x_{i}} \\
& =2 \Lambda\left(|\nabla u|^{2}\right) \sum_{k} \frac{\partial^{2} u}{\partial x_{i} \partial x_{k}} \frac{\partial u}{\partial x_{k}}-2 f(u) \frac{\partial u}{\partial x_{i}} .
\end{aligned}
$$

Hence, recalling (3.3), we get

$$
\begin{aligned}
& \sum_{i, j} \frac{\partial}{\partial x_{j}}\left(d_{i j}(\nabla u) \frac{\partial P}{\partial x_{i}}\right) \\
= & \sum_{i, j} \frac{\partial}{\partial x_{j}}\left(-2 f(u) d_{i j}(\nabla u) \frac{\partial u}{\partial x_{i}}+2 \Lambda\left(|\nabla u|^{2}\right) \frac{a_{i j}(\nabla u)}{\Lambda\left(|\nabla u|^{2}\right)} \sum_{k} \frac{\partial^{2} u}{\partial x_{i} \partial x_{k}} \frac{\partial u}{\partial x_{k}}\right) \\
= & -2 \sum_{i, j} \frac{\partial}{\partial x_{j}}\left(f(u) d_{i j}(\nabla u) \frac{\partial u}{\partial_{x_{i}}}\right)+2 \sum_{i, j, k} \frac{\partial}{\partial x_{j}}\left(a_{i j}(\nabla u) \frac{\partial^{2} u}{\partial x_{i} \partial x_{k}}\right) \frac{\partial u}{\partial x_{k}} \\
& +2 \sum_{i, j, k} a_{i j}(\nabla u) \frac{\partial^{2} u}{\partial x_{i} \partial x_{k}} \frac{\partial^{2} u}{\partial x_{j} \partial x_{k}} .
\end{aligned}
$$

Also, (1.3) gives that

$$
\frac{\partial a_{i j}}{\partial \sigma_{l}}(\sigma)=\frac{\partial a_{l j}}{\partial \sigma_{i}}(\sigma)
$$

By (1.1), we obtain

$$
\sum_{i, j} a_{i j}(\nabla u) \frac{\partial^{2} u}{\partial x_{i} \partial x_{j}}=f(u)+g(\nabla u, S u)
$$


Therefore, by (3.10) and (3.11), for any fixed $k$, we have

$$
\begin{aligned}
& \sum_{i, j} \frac{\partial}{\partial x_{j}}\left(a_{i j}(\nabla u) \frac{\partial^{2} u}{\partial x_{i} \partial x_{k}}\right) \\
= & \sum_{i, j}\left(\frac{\partial a_{i j}(\nabla u)}{\partial x_{j}} \frac{\partial^{2} u}{\partial x_{i} \partial x_{k}}+a_{i j}(\nabla u) \frac{\partial^{3} u}{\partial x_{i} \partial x_{k} \partial x_{j}}\right) \\
= & \sum_{i, j} \frac{\partial}{\partial x_{k}}\left(a_{i j}(\nabla u) \frac{\partial^{2} u}{\partial x_{i} \partial x_{j}}\right) \\
= & f^{\prime}(u) \frac{\partial u}{\partial x_{k}}+\sum_{j=1}^{n} g_{\zeta_{j}}(\nabla u, S u) \frac{\partial^{2} u}{\partial x_{j} \partial x_{k}}+\sum_{j=1}^{N-n} g_{\eta_{j}}(\nabla u, S u) \frac{\partial S^{[j]} u}{\partial x_{k}} .
\end{aligned}
$$

From (3.9) and (3.12), we find that

$$
\begin{aligned}
& \sum_{i, j} \frac{\partial}{\partial x_{j}}\left(d_{i j}(\nabla u) \frac{\partial P}{\partial x_{i}}\right) \\
= & -2 \sum_{i, j} f^{\prime}(u) d_{i j}(\nabla u) \frac{\partial u}{\partial x_{i}} \frac{\partial u}{\partial x_{j}}-2 f(u) \sum_{i, j} \frac{\partial}{\partial x_{j}}\left(d_{i j}(\nabla u) \frac{\partial u}{\partial x_{i}}\right) \\
& +2 f^{\prime}(u) \sum_{k} \frac{\partial u}{\partial x_{k}} \frac{\partial u}{\partial x_{k}}+2 \sum_{k}\left[\sum_{j=1}^{n} g_{\zeta_{j}}(\nabla u, S u) \frac{\partial^{2} u}{\partial x_{j} \partial x_{k}}+\sum_{j=1}^{N-n} g_{\eta_{j}}(\nabla u, S u) \frac{\partial S^{[j]} u}{\partial x_{k}}\right] \frac{\partial u}{\partial x_{k}} \\
& +2 \sum_{i, j, k} a_{i j}(\nabla u) \frac{\partial^{2} u}{\partial x_{i} \partial x_{k}} \frac{\partial^{2} u}{\partial x_{j} \partial x_{k}} .
\end{aligned}
$$

Furthermore, from (1.13) and (3.3), we obtain

$$
\begin{aligned}
& -\sum_{i, j} f^{\prime}(u) d_{i j}(\nabla u) \frac{\partial u}{\partial x_{i}} \frac{\partial u}{\partial x_{j}}+f^{\prime}(u) \sum_{k} \frac{\partial u}{\partial x_{k}} \frac{\partial u}{\partial x_{k}} \\
= & -\sum_{i, j} \frac{f^{\prime}(u)}{\Lambda\left(|\nabla u|^{2}\right)}\left[2 \Phi^{\prime \prime}\left(|\nabla u|^{2}\right)\left(\frac{\partial u}{\partial x_{i}}\right)^{2}\left(\frac{\partial u}{\partial x_{j}}\right)^{2}+\Phi^{\prime}\left(|\nabla u|^{2}\right) \delta_{i j} \frac{\partial u}{\partial x_{i}} \frac{\partial u}{\partial x_{j}}\right] \\
& +f^{\prime}(u) \sum_{k}\left(\frac{\partial u}{\partial x_{k}}\right)^{2} \\
= & -\frac{f^{\prime}(u)}{\Lambda\left(|\nabla u|^{2}\right)}\left[2 \Phi^{\prime \prime}\left(|\nabla u|^{2}\right)|\nabla u|^{4}+\Phi^{\prime}\left(|\nabla u|^{2}\right)|\nabla u|^{2}\right]+f^{\prime}(u)|\nabla u|^{2} \\
=0 . &
\end{aligned}
$$

Plugging this into (3.13), we conclude that

$$
\begin{aligned}
& \sum_{i, j} \frac{\partial}{\partial x_{j}}\left(d_{i j}(\nabla u) \frac{\partial P}{\partial x_{i}}\right) \\
= & -2 f(u) \sum_{i, j} \frac{\partial}{\partial x_{j}}\left(d_{i j}(\nabla u) \frac{\partial u}{\partial_{x_{i}}}\right)+2 \sum_{k}\left[\sum_{j=1}^{n} g_{\zeta_{j}}(\nabla u, S u) u_{j k}\right. \\
& \left.+\sum_{j=1}^{N-n} g_{\eta_{j}}(\nabla u, S u) \frac{\partial S^{[j]} u}{\partial x_{k}}\right] \frac{\partial u}{\partial x_{k}}+2 \sum_{i, j, k} a_{i j}(\nabla u) \frac{\partial^{2} u}{\partial x_{i} \partial x_{k}} \frac{\partial^{2} u}{\partial x_{j} \partial x_{k}} .
\end{aligned}
$$


Also, it follows from (3.3) and (3.11) that

$$
\sum_{i, j} d_{i j}(\nabla u) \frac{\partial^{2} u}{\partial x_{i} \partial x_{j}}=\frac{f(u)+g(\nabla u, S u)}{\Lambda\left(|\nabla u|^{2}\right)}
$$

and so (3.16) becomes

$$
\begin{aligned}
& \sum_{i, j} \frac{\partial}{\partial x_{j}}\left(d_{i j}(\nabla u) \frac{\partial P}{\partial x_{i}}\right) \\
= & -2 f(u) \sum_{i, j} \frac{\partial}{\partial x_{j}} d_{i j}(\nabla u) \frac{\partial u}{\partial x_{i}}-\frac{2 f(u)[f(u)+g(\nabla u, S u)]}{\Lambda\left(|\nabla u|^{2}\right)} \\
& +2 \sum_{k}\left[\sum_{j=1}^{n} g_{\zeta_{j}}(\nabla u, S u) u_{j k}+\sum_{j=1}^{N-n} g_{\eta_{j}}(\nabla u, S u) \frac{\partial S^{[j]} u}{\partial x_{k}}\right] \frac{\partial u}{\partial x_{k}} \\
& +2 \sum_{i, j, k} a_{i j}(\nabla u) \frac{\partial^{2} u}{\partial x_{i} \partial x_{k}} \frac{\partial^{2} u}{\partial x_{j} \partial x_{k}} .
\end{aligned}
$$

Moreover, making use of (1.3), (1.13) and (3.3), we obtain

$$
\begin{aligned}
& \sum_{i, j} \frac{\partial}{\partial x_{j}} d_{i j}(\nabla u) \frac{\partial u}{\partial x_{i}} \\
= & \sum_{i, j} \frac{\partial u}{\partial x_{i}} \frac{\partial}{\partial x_{j}} \frac{2 \Phi^{\prime \prime}\left(|\nabla u|^{2}\right) \frac{\partial u}{\partial x_{i}} \frac{\partial u}{\partial x_{j}}+\Phi^{\prime}\left(|\nabla u|^{2}\right) \delta_{i j}}{2 \Phi^{\prime \prime}\left(|\nabla u|^{2}\right)|\nabla u|^{2}+\Phi^{\prime}\left(|\nabla u|^{2}\right)} \\
& -\sum_{i, j} \frac{\partial u}{\partial x_{i}} \frac{\left[2 \Phi^{\prime \prime} \frac{\partial u}{\partial x_{i}} \frac{\partial u}{\partial x_{j}}+\Phi^{\prime} \delta_{i j}\right]\left[4 \Phi^{\prime \prime \prime}|\nabla u|^{2}+4 \Phi^{\prime \prime}+2 \Phi^{\prime \prime}\right] \sum_{k} \frac{\partial u}{\partial x_{k}} \frac{\partial^{2} u}{\partial x_{k} \partial x_{j}}}{\left[2 \Phi^{\prime \prime}\left(|\nabla u|^{2}\right)|\nabla u|^{2}+\Phi^{\prime}\left(|\nabla u|^{2}\right)\right]^{2}} \\
= & \frac{2 \Phi^{\prime \prime}\left[\Sigma_{j}|\nabla u|^{2} \frac{\partial^{2} u}{\partial x_{j}^{2}}-\sum_{i, j} \frac{\partial u}{\partial x_{i}} \frac{\partial^{2} u}{\partial x_{i} \partial x_{j}} \frac{\partial u}{\partial x_{j}}\right]}{2 \Phi^{\prime \prime}\left(|\nabla u|^{2}\right)|\nabla u|^{2}+\Phi^{\prime}\left(|\nabla u|^{2}\right)} \\
= & \frac{2 \Phi^{\prime \prime}\left(|\nabla u|^{2}\right)}{\Lambda\left(|\nabla u|^{2}\right)}\left(|\nabla u|^{2} \Delta u-\sum_{i, j} \frac{\partial^{2} u}{\partial x_{i} \partial x_{j}} \frac{\partial u}{\partial x_{i}} \frac{\partial u}{\partial x_{j}}\right) .
\end{aligned}
$$

Also, from (1.3) and (3.11), we get

$$
\begin{aligned}
f(u)+g(\nabla u, S u) & =\sum_{i, j}\left(2 \Phi^{\prime \prime}\left(|\nabla u|^{2}\right) \frac{\partial u}{\partial x_{i}} \frac{\partial u}{\partial x_{j}}+\Phi^{\prime}\left(|\nabla u|^{2}\right) \delta_{i j}\right) \frac{\partial^{2} u}{\partial x_{i} \partial x_{j}} \\
& =2 \Phi^{\prime \prime}\left(|\nabla u|^{2}\right) \sum_{i, j} \frac{\partial^{2} u}{\partial x_{i} \partial x_{j}} \frac{\partial u}{\partial x_{i}} \frac{\partial u}{\partial x_{j}}+\Phi^{\prime}\left(|\nabla u|^{2}\right) \Delta u
\end{aligned}
$$

from which we obtain

$$
\Delta u=\frac{f(u)+g(\nabla u, S u)}{\Phi^{\prime}\left(|\nabla u|^{2}\right)}-2 \frac{\Phi^{\prime \prime}\left(|\nabla u|^{2}\right)}{\Phi^{\prime}\left(|\nabla u|^{2}\right)} \sum_{i, j} \frac{\partial^{2} u}{\partial x_{i} \partial x_{j}} \frac{\partial u}{\partial x_{i}} \frac{\partial u}{\partial x_{j}} .
$$

Therefore, recalling also (3.8), we write (3.17) as

$$
\sum_{i, j} \frac{\partial}{\partial x_{j}} d_{i j}(\nabla u) \frac{\partial u}{\partial x_{i}}=\frac{2 \Phi^{\prime \prime}\left(|\nabla u|^{2}\right)}{\Lambda\left(|\nabla u|^{2}\right) \Phi^{\prime}\left(|\nabla u|^{2}\right)}\left[|\nabla u|^{2}(f+g)-\Lambda\left(|\nabla u|^{2}\right) \sum_{i, j} \frac{\partial^{2} u}{\partial x_{i} \partial x_{j}} \frac{\partial u}{\partial x_{i}} \frac{\partial u}{\partial x_{j}}\right]
$$




$$
=\frac{-\Phi^{\prime \prime}\left(|\nabla u|^{2}\right)}{\Lambda\left(|\nabla u|^{2}\right) \Phi^{\prime}\left(|\nabla u|^{2}\right)} \sum_{i} \frac{\partial P}{\partial x_{i}} \frac{\partial u}{\partial x_{i}}+\frac{2 g \Phi^{\prime \prime}\left(|\nabla u|^{2}\right)|\nabla u|^{2}}{\Lambda\left(|\nabla u|^{2}\right) \Phi^{\prime}\left(|\nabla u|^{2}\right)} .
$$

Thus, exploiting (3.16), one has

$$
\begin{aligned}
& \sum_{i, j} \frac{\partial}{\partial x_{j}}\left(d_{i j}(\nabla u) \frac{\partial P}{\partial x_{i}}\right) \\
= & \frac{2 f(u) \Phi^{\prime \prime}\left(|\nabla u|^{2}\right)}{\Lambda\left(|\nabla u|^{2}\right) \Phi^{\prime}\left(|\nabla u|^{2}\right)} \sum_{i} \frac{\partial P}{\partial x_{i}} \frac{\partial u}{\partial x_{i}}-\frac{4 f g \Phi^{\prime \prime}\left(|\nabla u|^{2}\right)|\nabla u|^{2}}{\Lambda\left(|\nabla u|^{2}\right) \Phi^{\prime}\left(|\nabla u|^{2}\right)}-\frac{2 f(u)[f(u)+g(\nabla u, S u)]}{\Lambda\left(|\nabla u|^{2}\right)} \\
& +2 \sum_{k}\left[\sum_{j=1}^{n} g_{\zeta_{j}}(\nabla u, S u) u_{j k}+\sum_{j=1}^{N-n} g_{\eta_{j}}(\nabla u, S u) \frac{\partial S^{[j]} u}{\partial x_{k}}\right] \frac{\partial u}{\partial x_{k}} \\
& +2 \sum_{i, j, k} a_{i j}(\nabla u) \frac{\partial^{2} u}{\partial x_{i} \partial x_{k}} \frac{\partial^{2} u}{\partial x_{j} \partial x_{k}} .
\end{aligned}
$$

Now we set

$$
z_{k}=\sum_{i} \frac{\partial^{2} u}{\partial x_{i} \partial x_{k}} \frac{\partial u}{\partial x_{i}}
$$

and we use Schwarz Inequality to see that

$$
\left|z_{k}\right| \leq \sqrt{\sum_{i}\left(\frac{\partial^{2} u}{\partial x_{i} \partial x_{k}}\right)^{2}} \sqrt{\sum_{i}\left(\frac{\partial u}{\partial x_{i}}\right)^{2}}
$$

and so

$$
\begin{aligned}
\sum_{i, j, k} \frac{\partial^{2} u}{\partial x_{i} \partial x_{k}} \frac{\partial u}{\partial x_{i}} \frac{\partial^{2} u}{\partial x_{j} \partial x_{k}} \frac{\partial u}{\partial x_{j}} & =\sum_{k}\left|z_{k}\right|^{2} \leq \sum_{k}\left(\sum_{i}\left(\frac{\partial^{2} u}{\partial x_{i} \partial x_{k}}\right)^{2}\right)\left(\sum_{i}\left(\frac{\partial u}{\partial x_{i}}\right)^{2}\right) \\
& =|\nabla u|^{2} \sum_{i, k}\left(\frac{\partial^{2} u}{\partial x_{i} \partial x_{k}}\right)^{2} .
\end{aligned}
$$

This and (1.3) give that

$$
\begin{aligned}
& \sum_{i, j, k} a_{i j}(\nabla u) \frac{\partial^{2} u}{\partial x_{i} \partial x_{k}} \frac{\partial^{2} u}{\partial x_{j} \partial x_{k}} \\
= & \sum_{i, j, k} 2 \Phi^{\prime \prime}\left(|\nabla u|^{2}\right) \frac{\partial^{2} u}{\partial x_{i} \partial x_{k}} \frac{\partial^{2} u}{\partial x_{j} \partial x_{k}} \frac{\partial u}{\partial x_{i}} \frac{\partial u}{\partial x_{j}}+\sum_{i, j, k} \Phi^{\prime}\left(|\nabla u|^{2}\right) \frac{\partial^{2} u}{\partial x_{i} \partial x_{k}} \frac{\partial^{2} u}{\partial x_{j} \partial x_{k}} \delta_{i j} \\
\geq & \sum_{i, j, k} 2 \Phi^{\prime \prime}\left(|\nabla u|^{2}\right) \frac{\partial^{2} u}{\partial x_{i} \partial x_{k}} \frac{\partial u}{\partial x_{i}} \frac{\partial^{2} u}{\partial x_{j} \partial x_{k}} \frac{\partial u}{\partial x_{j}}+\sum_{i, j, k} \frac{\Phi^{\prime}\left(|\nabla u|^{2}\right)}{|\nabla u|^{2}} \frac{\partial^{2} u}{\partial x_{i} \partial x_{k}} \frac{\partial u}{\partial x_{i}} \frac{\partial^{2} u}{\partial x_{j} \partial x_{k}} \frac{\partial u}{\partial x_{j}} \\
= & \frac{\Lambda\left(|\nabla u|^{2}\right)}{|\nabla u|^{2}} \sum_{i, j, k} \frac{\partial^{2} u}{\partial x_{i} \partial x_{k}} \frac{\partial u}{\partial x_{i}} \frac{\partial^{2} u}{\partial x_{j} \partial x_{k}} \frac{\partial u}{\partial x_{j}} .
\end{aligned}
$$

Moreover, by (3.8), we have that

$$
\sum_{i, j, k} \frac{\partial^{2} u}{\partial x_{i} \partial x_{k}} \frac{\partial u}{\partial x_{i}} \frac{\partial^{2} u}{\partial x_{j} \partial x_{k}} \frac{\partial u}{\partial x_{j}}=\frac{1}{4 \Lambda^{2}\left(|\nabla u|^{2}\right)} \sum_{k}\left(\frac{\partial P}{\partial x_{k}}+2 f \frac{\partial u}{\partial x_{k}}\right)^{2} .
$$


This and (3.20) lead to

$$
\begin{aligned}
\sum_{i, j, k} a_{i j}(\nabla u) \frac{\partial^{2} u}{\partial x_{i} \partial x_{k}} \frac{\partial^{2} u}{\partial x_{j} \partial x_{k}} & \geq \frac{1}{4 \Lambda\left(|\nabla u|^{2}\right)|\nabla u|^{2}} \sum_{k}\left(\frac{\partial P}{\partial x_{k}}+2 f \frac{\partial u}{\partial x_{k}}\right)^{2} \\
& =\frac{|\nabla P|^{2}}{4 \Lambda\left(|\nabla u|^{2}\right)|\nabla u|^{2}}+\frac{f \sum_{k} \frac{\partial P}{\partial x_{k}} \frac{\partial u}{\partial x_{k}}}{\Lambda\left(|\nabla u|^{2}\right)|\nabla u|^{2}}+\frac{f^{2}}{\Lambda\left(|\nabla u|^{2}\right)}
\end{aligned}
$$

By substituting this into (3.19), we obtain

$$
\begin{aligned}
& \sum_{i, j} \frac{\partial}{\partial x_{j}}\left(d_{i j}(\nabla u) \frac{\partial P}{\partial x_{i}}\right) \\
\geq & \frac{2 f(u) \Phi^{\prime \prime}\left(|\nabla u|^{2}\right)}{\Lambda\left(|\nabla u|^{2}\right) \Phi^{\prime}\left(|\nabla u|^{2}\right)} \sum_{i} \frac{\partial P}{\partial x_{i}} \frac{\partial u}{\partial x_{i}}-\frac{4 f g \Phi^{\prime \prime}\left(|\nabla u|^{2}\right)|\nabla u|^{2}}{\Lambda\left(|\nabla u|^{2}\right) \Phi^{\prime}\left(|\nabla u|^{2}\right)}-\frac{2 f(u)[f(u)+g(\nabla u, S u)]}{\Lambda\left(|\nabla u|^{2}\right)} \\
& +2 \sum_{k}\left[\sum_{j=1}^{n} g_{\zeta_{j}}(\nabla u, S u) \frac{\partial^{2} u}{\partial x_{j} \partial x_{k}}+\sum_{j=1}^{N-n} g_{\eta_{j}}(\nabla u, S u) \frac{\partial S^{[j]} u}{\partial x_{k}}\right] \frac{\partial u}{\partial x_{k}}+\frac{|\nabla P|^{2}}{2 \Lambda\left(|\nabla u|^{2}\right)|\nabla u|^{2}} \\
& +2 \frac{f \sum_{k} \frac{\partial P}{\partial x_{k}} \frac{\partial u}{\partial x_{k}}}{\Lambda\left(|\nabla u|^{2}\right)|\nabla u|^{2}}+2 \frac{f^{2}}{\Lambda\left(|\nabla u|^{2}\right)} .
\end{aligned}
$$

Therefore, we have that

$$
\begin{aligned}
& \sum_{i, j} \frac{\partial}{\partial x_{j}}\left(d_{i j}(\nabla u) \frac{\partial P}{\partial x_{i}}\right)-2 \frac{f(u)}{\Lambda\left(|\nabla u|^{2}\right)|\nabla u|^{2}}\left(1+\frac{\Phi^{\prime \prime}\left(|\nabla u|^{2}\right)|\nabla u|^{2}}{\Phi^{\prime}\left(|\nabla u|^{2}\right)}\right) \sum_{k} \frac{\partial P}{\partial x_{k}} \frac{\partial u}{\partial x_{k}} \\
\geq & -\frac{4 f g \Phi^{\prime \prime}\left(|\nabla u|^{2}\right)|\nabla u|^{2}}{\Lambda\left(|\nabla u|^{2}\right) \Phi^{\prime}\left(|\nabla u|^{2}\right)}-\frac{2 f(u) g(\nabla u, S u)}{\Lambda\left(|\nabla u|^{2}\right)} \\
& +2 \sum_{k}\left[\sum_{j=1}^{n} g_{\zeta_{j}}(\nabla u, S u) \frac{\partial^{2} u}{\partial x_{j} \partial x_{k}}+\sum_{j=1}^{N-n} g_{\eta_{j}}(\nabla u, S u) \frac{\partial S^{[j]} u}{\partial x_{k}}\right] \frac{\partial u}{\partial x_{k}}+\frac{|\nabla P|^{2}}{2 \Lambda\left(|\nabla u|^{2}\right)|\nabla u|^{2}} \\
= & -\frac{2 f(u) g(\nabla u, S u)}{\Phi^{\prime}\left(|\nabla u|^{2}\right)}+2 \sum_{k}\left[\sum_{j=1}^{n} g_{\zeta_{j}}(\nabla u, S u) \frac{\partial^{2} u}{\partial x_{j} \partial x_{k}}+\sum_{j=1}^{N-n} g_{\eta_{j}}(\nabla u, S u) \frac{\partial S^{[j]} u}{\partial x_{k}}\right] \frac{\partial u}{\partial x_{k}} \\
& +\frac{|\nabla P|^{2}}{2 \Lambda\left(|\nabla u|^{2}\right)|\nabla u|^{2}} .
\end{aligned}
$$

Now, for $j$ fixed, we use (3.6) to get that

$$
2 \sum_{k} \frac{\partial^{2} u}{\partial x_{j} \partial x_{k}} \frac{\partial u}{\partial x_{k}}=\frac{\partial|\nabla u(x)|^{2}}{\partial x_{j}}=\Psi^{\prime}(P+2 F)\left(\frac{\partial P}{\partial x_{j}}+2 f(u) \frac{\partial u}{\partial x_{j}}\right) .
$$

Consequently, by (3.7), we conclude

$$
\begin{aligned}
2 \sum_{k, j} g_{\zeta_{j}}(\nabla u, S u) \frac{\partial^{2} u}{\partial x_{j} \partial x_{k}} \frac{\partial u}{\partial x_{k}} & =\Psi^{\prime}(P+2 F) \sum_{j} g_{\zeta_{j}}(\nabla u, S u)\left(\frac{\partial P}{\partial x_{j}}+2 f(u) \frac{\partial u}{\partial x_{j}}\right) \\
& =\frac{1}{\Lambda\left(|u(x)|^{2}\right)} \sum_{j} g_{\zeta_{j}}(\nabla u, S u)\left(\frac{\partial P}{\partial x_{j}}+2 f(u) \frac{\partial u}{\partial x_{j}}\right) .
\end{aligned}
$$


Multiplying both sides of (3.21) by $|\nabla u|^{2}$, we see that

$$
\begin{aligned}
& \sum_{i, j}|\nabla u|^{2} \frac{\partial}{\partial x_{j}}\left(d_{i j}(\nabla u) \frac{\partial P}{\partial x_{i}}\right)-2 \frac{f(u)}{\Lambda\left(|\nabla u|^{2}\right)}\left(1+\frac{\Phi^{\prime \prime}\left(|\nabla u|^{2}\right)|\nabla u|^{2}}{\Phi^{\prime}\left(|\nabla u|^{2}\right)}\right) \sum_{k} \frac{\partial P}{\partial x_{k}} \frac{\partial u}{\partial x_{k}} \\
\geq & -\frac{2 f(u) g(\nabla u, S u)|\nabla u|^{2}}{\Phi^{\prime}\left(|\nabla u|^{2}\right)}+\frac{|\nabla P|^{2}}{2 \Lambda\left(|\nabla u|^{2}\right)} \\
& +2|\nabla u|^{2} \sum_{k}\left[\sum_{j=1}^{n} g_{\zeta_{j}}(\nabla u, S u) \frac{\partial^{2} u}{\partial x_{j} \partial x_{k}}+\sum_{j=1}^{N-n} g_{\eta_{j}}(\nabla u, S u) \frac{\partial S^{[j]} u}{\partial x_{k}}\right] \frac{\partial u}{\partial x_{k}} \\
= & -\frac{2 f(u) g(\nabla u, S u)|\nabla u|^{2}}{\Phi^{\prime}\left(|\nabla u|^{2}\right)}+2|\nabla u|^{2} \sum_{k=1}^{n} \sum_{j=1}^{N-n} g_{\eta_{j}}(\nabla u, S u) \frac{\partial S^{[j]} u}{\partial x_{k}} \frac{\partial u}{\partial x_{k}} \\
& +\frac{|\nabla u|^{2}}{\Lambda\left(|u(x)|^{2}\right)} \sum_{j} g_{\zeta_{j}}(\nabla u, S u)\left(\frac{\partial P}{\partial x_{j}}+2 f(u) \frac{\partial u}{\partial x_{j}}\right)+\frac{|\nabla P|^{2}}{2 \Lambda\left(|\nabla u|^{2}\right)} \\
= & \mathscr{R}(x)+\frac{|\nabla u|^{2}}{\Lambda\left(|u(x)|^{2}\right)} \sum_{j} g_{\zeta_{j}}(\nabla u, S u) \frac{\partial P}{\partial x_{j}}+\frac{|\nabla P|^{2}}{2 \Lambda\left(|\nabla u|^{2}\right)} .
\end{aligned}
$$

From this, we obtain the desired result in (3.5).

\section{Proof of Theorem 1.2}

This section contains the proof of the pointwise gradient estimate in (1.18). This relies on Lemma 3.1 and the Maximum Principle. The technical details go as follows:

Proof of Theorem 1.2. First of all, we observe that (3.2) holds true. Indeed, taking $\xi:=(1,0, \ldots, 0)$ and $\sigma:=\sqrt{r} \xi$, we deduce from (1.3) and (1.13) that

$$
\sum_{i, j=1}^{n} a_{i j}(\sigma) \xi_{i} \xi_{j}=2 \Phi^{\prime \prime}\left(|\sigma|^{2}\right)(\sigma \cdot \xi)^{2}+\Phi^{\prime}\left(|\sigma|^{2}\right)|\xi|^{2}=2 \Phi^{\prime \prime}(r) r+\Phi^{\prime}(r)=\Lambda(r) .
$$

Hence, if Assumption A is satisfied, we obtain

$$
\Lambda(r)=\sum_{i, j=1}^{n} a_{i j}(\sigma) \xi_{i} \xi_{j} \geq C_{1}(a+|\sigma|)^{p-2}|\xi|^{2}=C_{1}(a+\sqrt{r})^{p-2}>0 .
$$

If instead Assumption B is satisfied, we deduce from (4.1) that

$$
\begin{gathered}
\Lambda(r)=\sum_{i, j=1}^{n} a_{i j}(\sigma) \xi_{i} \xi_{j} \geq C_{1}(1+|\sigma|)^{-1}\left|\xi^{\prime}\right|^{2}=C_{1}(1+|\sigma|)^{-1}\left(|\xi|^{2}+(\sigma \cdot \xi)^{2}\right) \\
=C_{1}(1+\sqrt{r})^{-1}(1+r)>0 .
\end{gathered}
$$

This observation and (4.2) show that (3.2) is satisfied, and therefore we are in the position of applying Lemma 3.1. In this way, recalling (1.17) and (3.5), we see

$$
\sum_{i, j} \frac{\partial}{\partial x_{j}}\left(d_{i j}(\nabla u(x)) \frac{\partial P(u ; x)}{\partial x_{i}}\right)+\frac{B(u ; x) \cdot \nabla P(u ; x)}{|\nabla u(x)|^{2}} \geq 0
$$


in $\{\nabla u \neq 0\}$, where the notations in (3.1) and (3.4) have been utilized.

From this, we can repeat some classical arguments used also in the proof of Theorem 1.2 in [FV13] to obtain our Theorem 1.2. We show the arguments in full detail for the facility of the reader. Besides, in order to address the general case treated in this paper, these classical arguments need to be carefully adapted, producing a number of additional technical difficulties.

Recalling the notation in (3.1), we define

$$
P_{0}:=\sup _{x \in \mathbb{R}^{n}} P(u ; x)
$$

We claim that

$$
P_{0} \leq 0
$$

To prove this, we assume by contradiction that

$$
P_{0}>0
$$

First, take sequence $z_{k} \in \mathbb{R}^{n}$ such that

$$
\lim _{k \rightarrow+\infty} P\left(u ; z_{k}\right)=P_{0} .
$$

We can define $w_{k}(x)=u\left(x+z_{k}\right)$. This function satisfies an elliptic equation with bounded right hand side and therefore, by elliptic regularity theory (possibly reducing Assumption B to Assumption A with $p=2$ ), we have that, for every $R>0$,

$$
\left\|w_{k}\right\|_{C^{1, \gamma}\left(B_{R}\right)}<+\infty
$$

for some $\gamma \in(0,1)$.

Also, from (4.5), we have that

$$
P_{0} \geq P\left(w_{k} ; x\right), \quad \text { for all } x \in \mathbb{R}^{n} .
$$

Furthermore, by (3.1), we get

$$
\begin{aligned}
& P\left(u ; z_{k}\right)=2 \Phi^{\prime}\left(\left|\nabla u\left(z_{k}\right)\right|^{2}\right)\left|\nabla u\left(z_{k}\right)\right|^{2}-\Phi\left(\left|\nabla u\left(z_{k}\right)\right|^{2}\right)-2 F\left(u\left(z_{k}\right)\right) \\
& \quad=2 \Phi^{\prime}\left(\left|\nabla w_{k}(0)\right|^{2}\right)\left|\nabla w_{k}(0)\right|^{2}-\Phi\left(\left|\nabla w_{k}(0)\right|^{2}\right)-2 F\left(w_{k}(0)\right)=P\left(w_{k} ; 0\right)
\end{aligned}
$$

In view of this and (4.8), we conclude that

$$
\lim _{k \rightarrow+\infty} P\left(w_{k} ; 0\right)=P_{0}
$$

By the Theorem of Ascoli-Arzelà (and up to a subsequence) and possibly renaming $\gamma$, we may suppose that $w_{k}$ converges to some $w$ in $C_{\text {loc }}^{1, \gamma}\left(\mathbb{R}^{n}\right)$, and therefore, by (3.1), we see

$$
\lim _{k \rightarrow+\infty} P\left(w_{k} ; x\right)=P(w ; x), \quad \text { for all } x \in \mathbb{R}^{n} .
$$

Using this, (4.10) and (4.11), we thereby obtain

$$
P(w ; x)=\lim _{k \rightarrow+\infty} P\left(w_{k} ; x\right) \leq P_{0}=\lim _{k \rightarrow+\infty} P\left(w_{k} ; 0\right)=P(w ; 0) .
$$

Now, we define

$$
\mathcal{N}:=\left\{x \in \mathbb{R}^{n} \text { s.t. } P(w ; x)=P_{0}\right\}
$$


We observe that $0 \in \mathcal{N}$, thanks to (4.12), and hence

$$
\mathcal{N} \neq \varnothing
$$

Also, by the continuity of $P$ and $w$,

$$
\mathcal{N} \text { is closed. }
$$

Here, we denote $\Psi^{-1}$ by $\Gamma$ for simplicity, so

$$
\Gamma(r):=2 \Phi^{\prime}(r) r-\Phi(r)
$$

and we claim that for all $r \in\left[0,\|u\|_{W^{1, \infty}\left(\mathbb{R}^{n}\right)}\right]$

$$
\Gamma(r) \leq C \sqrt{r} .
$$

To prove this, we first remark that

$$
\Gamma(0)=0,
$$

by $\Phi(0)=0$ and either (1.4) or (1.6). Moreover, taking $\sigma:=\sqrt{r} e_{1}, \xi:=e_{1}, \xi_{n+1}:=\sqrt{r}$, by (1.3) we see that

$$
a_{i j}(\sigma) \xi_{i} \xi_{j}= \begin{cases}2 \Phi^{\prime \prime}(r) r+\Phi^{\prime}(r)=\Gamma^{\prime}(r) & \text { if } i=j=1 \\ 0 & \text { otherwise }\end{cases}
$$

and accordingly

$$
\sum_{i, j=1}^{n} a_{i j}(\sigma) \xi_{i} \xi_{j}=\Gamma^{\prime}(r)
$$

Now, to prove (4.16) we distinguish two cases, according to whether Assumption A or Assumption B is satisfied. First of all, if Assumption A is satisfied, we use (1.5) and (4.18) to see that

$$
\Gamma^{\prime}(r) \leq C_{2}(a+|\sigma|)^{p-2}|\xi|^{2}=C_{2}(a+\sqrt{r})^{p-2} .
$$

We now distinguish two subcases, depending on $p$. If $p \geq 2$, we deduce from (4.19) that

$$
\Gamma^{\prime}(r) \leq C\left(1+r^{\frac{p-2}{2}}\right)
$$

for some $C>0$. This and (4.17) yield that

$$
\begin{gathered}
\Gamma(r)=\int_{0}^{r} \Gamma^{\prime}(\rho) d \rho \leq C\left(r+\frac{2}{p} r^{\frac{p}{2}}\right)=C\left(\sqrt{r}+\frac{2}{p} r^{\frac{p-1}{2}}\right) \sqrt{r} \\
\leq C\left(\sqrt{\|u\|_{W^{1, \infty}\left(\mathbb{R}^{n}\right)}}+\frac{2}{p}\|u\|_{W^{1, \infty}\left(\mathbb{R}^{n}\right)}^{\frac{p-1}{2}}\right) \sqrt{r},
\end{gathered}
$$

for all $r \in\left[0,\|u\|_{W^{1, \infty}\left(\mathbb{R}^{n}\right)}\right]$, and this gives (4.16), up to renaming $C>0$.

On the other hand, if $p \in(1,2)$, we deduce from (4.19) that

$$
\Gamma^{\prime}(r) \leq \frac{C_{2}}{(a+\sqrt{r})^{2-p}} \leq \frac{C_{2}}{r^{\frac{2-p}{2}}},
$$

which, together with (4.17), gives that

$$
\Gamma(r)=\int_{0}^{r} \Gamma^{\prime}(\rho) d \rho \leq \frac{2 C_{2} r^{\frac{p}{2}}}{p} \leq \frac{2 C_{2}\|u\|_{W^{1, \infty}\left(\mathbb{R}^{n}\right)}^{\frac{p-1}{2}}}{p} \sqrt{r},
$$


for all $r \in\left[0,\|u\|_{W^{1, \infty}\left(\mathbb{R}^{n}\right)}\right]$, and this gives (4.16).

It remains to prove (4.16) if Assumption B holds true. In this case, we use (1.7) and (4.18) to see that

$$
\Gamma^{\prime}(r) \leq C_{2}(1+|\sigma|)^{-1}\left|\xi^{\prime}\right|^{2}=C_{2}(1+|\sigma|)^{-1}\left(|\xi|^{2}+\left|\xi_{n+1}\right|^{2}\right)=C_{2}(1+\sqrt{r})^{-1}(1+r) \leq C_{2}(1+r) .
$$

This and (4.17) give that

$$
\Gamma(r) \leq C_{2}\left(r+\frac{r^{2}}{2}\right) \leq C_{2}\left(\sqrt{\|u\|_{W^{1, \infty}\left(\mathbb{R}^{n}\right)}}+\frac{\|u\|_{W^{1, \infty}\left(\mathbb{R}^{n}\right)}^{\frac{3}{2}}}{2}\right) \sqrt{r}
$$

for all $r \in\left[0,\|u\|_{W^{1, \infty}\left(\mathbb{R}^{n}\right)}\right]$, and the proof of (4.16) is thereby complete.

Now, we claim that

$$
\mathcal{N} \text { is open. }
$$

For this, let $y_{0} \in \mathcal{N}$. We recall that $F \geq 0$ on the range of $u$, thanks to (1.15). Then, in light of (3.1), (4.15) and (4.16) we see that

$$
\begin{aligned}
P_{0}= & P\left(w ; y_{0}\right)=2 \Phi^{\prime}\left(\left|\nabla w\left(y_{0}\right)\right|^{2}\right)\left|\nabla w\left(y_{0}\right)\right|^{2}-\Phi\left(\left|\nabla w\left(y_{0}\right)\right|^{2}\right)-2 F\left(w\left(y_{0}\right)\right) \\
& =\Gamma\left(\left|\nabla w\left(y_{0}\right)\right|^{2}\right)-2 F\left(w\left(y_{0}\right)\right) \leq \Gamma\left(\left|\nabla w\left(y_{0}\right)\right|^{2}\right) \leq C\left|\nabla w\left(y_{0}\right)\right| .
\end{aligned}
$$

Now, we set

$$
\kappa:=\frac{P_{0}}{2 C}
$$

and, recalling (4.7), we observe that $\kappa>0$. As a consequence, in light of (4.21), it follows that there exists $\varrho>0$ such that

$$
|\nabla w(x)| \geq \kappa, \quad \text { for any } x \in B_{\varrho}\left(y_{0}\right) .
$$

Therefore, there exists $\bar{k} \in \mathbb{N}$ such that for all $k \geq \bar{k}$ and all $x \in B_{\varrho}\left(y_{0}\right)$ we have that

$$
\left|\nabla u\left(x+z_{k}\right)\right|=\left|\nabla w_{k}(x)\right| \geq \frac{\kappa}{2} .
$$

In particular, $\nabla u\left(x+z_{k}\right) \neq 0$ and therefore, by (4.4), we get

$$
\sum_{i, j} \frac{\partial}{\partial x_{j}}\left(d_{i j}\left(\nabla u\left(x+z_{k}\right)\right) \frac{\partial P\left(u ; x+z_{k}\right)}{\partial x_{i}}\right)+\frac{B\left(u ; x+z_{k}\right) \cdot \nabla P\left(u ; x+z_{k}\right)}{\left|\nabla u\left(x+z_{k}\right)\right|^{2}} \geq 0 .
$$

Moreover, by (3.1), we have $P\left(u ; x+z_{k}\right)=P\left(w_{k} ; x\right)$, and therefore we can write (4.23) in the form

$$
\begin{aligned}
0 & \leq \sum_{i, j} \frac{\partial}{\partial x_{j}}\left(d_{i j}\left(\nabla w_{k}(x)\right) \frac{\partial P\left(w_{k} ; x\right)}{\partial x_{i}}\right)+\frac{B\left(u ; x+z_{k}\right) \cdot \nabla P\left(w_{k} ; x\right)}{\left|\nabla u\left(x+z_{k}\right)\right|^{2}} \\
& =\sum_{i, j} \frac{\partial}{\partial x_{j}}\left(d_{i j}\left(\nabla w_{k}(x)\right) \frac{\partial P\left(w_{k} ; x\right)}{\partial x_{i}}\right)+\beta_{k}(x) \cdot \nabla P\left(w_{k} ; x\right),
\end{aligned}
$$

for all $x \in B_{\varrho}\left(y_{0}\right)$, as long as $k \geq \bar{k}$, where

$$
\beta_{k}(x):=\frac{B\left(u ; x+z_{k}\right)}{\left|\nabla u\left(x+z_{k}\right)\right|^{2}} .
$$


We stress that, by (3.4), (4.22) and (4.25), we can obtain

$$
\begin{aligned}
& \sup \left\|\beta_{k}\right\|_{L^{\infty}\left(B_{\varrho}\left(y_{0}\right), \mathbb{R}^{n}\right)} \\
& \leq \frac{4}{\kappa^{2}}\left[\frac{2\|f\|_{L^{\infty}\left(u\left(\mathbb{R}^{n}\right)\right)}}{\inf _{\kappa / 2 \leq|\zeta| \leq\|u\|_{W^{1, \infty}\left(\mathbb{R}^{n}\right)}} \Lambda\left(|\zeta|^{2}\right)}\left(1+\sup _{\kappa / 2 \leq|\zeta| \leq\|u\|_{W^{1, \infty}\left(\mathbb{R}^{n}\right)}} \frac{|\zeta|^{2} \Phi^{\prime \prime}\left(|\zeta|^{2}\right)}{\Phi^{\prime}\left(|\zeta|^{2}\right)}\right)\|u\|_{W^{1, \infty}\left(\mathbb{R}^{n}\right)}\right.
\end{aligned}
$$

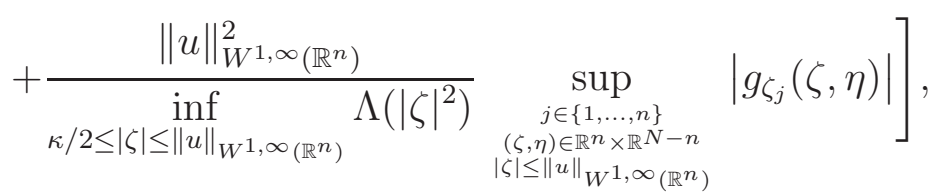

which is bounded, thanks to (1.2) (recall also (4.2) and (4.3)). Therefore, up to subsequences, we can suppose that

$$
\begin{aligned}
& \beta_{k} \text { converges to some } \beta \in L^{\infty}\left(B_{\varrho}\left(y_{0}\right), \mathbb{R}^{n}\right) \\
& \text { weakly in } L^{2}\left(B_{\varrho}\left(y_{0}\right), \mathbb{R}^{n}\right) \text { and weakly-* in } L^{\infty}\left(B_{\varrho}\left(y_{0}\right), \mathbb{R}^{n}\right) .
\end{aligned}
$$

Furthermore, by (1.1), we conclude

$$
\begin{aligned}
0 & =\operatorname{div}\left(\Phi^{\prime}\left(\left|\nabla u\left(x+z_{k}\right)\right|^{2}\right) \nabla u\left(x+z_{k}\right)\right)-f\left(u\left(x+z_{k}\right)\right)-g\left(\nabla u\left(x+z_{k}\right), S u\left(x+z_{k}\right)\right) \\
& =\operatorname{div}\left(\Phi^{\prime}\left(\left|\nabla w_{k}(x)\right|^{2}\right) \nabla w_{k}(x)\right)-f\left(w_{k}(x)\right)-g\left(\nabla w_{k}(x), S u\left(x+z_{k}\right)\right) \\
& =\operatorname{div}\left(\Phi^{\prime}\left(\left|\nabla w_{k}(x)\right|^{2}\right) \nabla w_{k}(x)\right)-\tilde{f}_{k}(x)
\end{aligned}
$$

where

$$
\tilde{f}_{k}(x):=f\left(w_{k}(x)\right)+g\left(\nabla w_{k}(x), S u\left(x+z_{k}\right)\right) .
$$

In view of (1.16) and (4.9), we have that $\tilde{f}_{k} \in C^{0, \gamma}\left(B_{\varrho}\left(y_{0}\right)\right)$, with

$$
\sup _{k \geq \bar{k}}\left\|\tilde{f}_{k}\right\|_{C^{0, \gamma}\left(B_{\varrho}\left(y_{0}\right)\right)}<+\infty
$$

Consequently, by (4.22) and uniform elliptic regularity theory, we obtain that

$$
\sup _{k \geq \bar{k}}\left\|w_{k}\right\|_{C^{2, \gamma}\left(B_{\varrho}\left(y_{0}\right)\right)}<+\infty .
$$

Therefore, up to a subsequence and possibly renaming $\gamma$, we can suppose that $w_{k}$ converges to $w$ in $C^{2, \gamma}\left(B_{\varrho}\left(y_{0}\right)\right)$, as $k \rightarrow+\infty$.

As a consequence, recalling (3.1), we conclude that

$$
\nabla P\left(w_{k} ; \cdot\right) \text { converges to } \nabla P(w ; \cdot) \text { in } C^{0, \gamma}\left(B_{\varrho}\left(y_{0}\right), \mathbb{R}^{n}\right) \text {, as } k \rightarrow+\infty \text {. }
$$

Exploiting (4.24), (4.26) and (4.27), we obtain that

$$
0 \leq \sum_{i, j} \frac{\partial}{\partial x_{j}}\left(d_{i j}(\nabla w(x)) \frac{\partial P(w ; x)}{\partial x_{i}}\right)+\beta(x) \cdot \nabla P(w ; x),
$$

for all $x \in B_{\varrho}\left(y_{0}\right)$, in the distributional sense.

Therefore, recalling (4.12), by Maximum Principle (see e.g. GT83, Theorem 8.19], or [PS07]), it follows that $P(w ; x)=P_{0}$ for any $x \in B_{\varrho}\left(y_{0}\right)$, and this establishes (4.20). 
Now, by (4.13) and (4.20), we infer that $\mathcal{N}$ is both closed and open, so that $\mathcal{N}=\mathbb{R}^{n}$, that is

$$
P(w ; x)=P_{0} \text { for any } x \in \mathbb{R}^{n} \text {. }
$$

On the other hand, since $w$ is bounded, by following the gradient lines we find a sequence of points $\tau_{j}$ such that

$$
\lim _{j \rightarrow+\infty} \nabla w\left(\tau_{j}\right)=0
$$

By using this in (4.28), we obtain

$$
0 \geq \limsup _{j \rightarrow+\infty}-2 F\left(w\left(\tau_{j}\right)\right)=\limsup _{j \rightarrow+\infty} P\left(w ; \tau_{j}\right)=P_{0},
$$

which is in contradiction with (4.7). This proves (4.6), from which Theorem 1.2 follows at once.

\section{Proofs of Proposition 1.3 and Corollary 1.4}

We start by proving Proposition 1.3:

Proof of Proposition 1.3. From the definition of $\Phi(r)$ in (1.8) and $\Lambda$ in (1.13) (recall also (2.7) and (2.15) ) we have that

$$
\begin{aligned}
& \Lambda(r)=2 r \Phi^{\prime \prime}(r)+\Phi^{\prime}(r)=\sum_{k=1}^{m}\left[c_{k}\left(p_{k}-2\right) r\left(b_{k}+r\right)^{\frac{p_{k}-4}{2}}+c_{k}\left(b_{k}+r\right)^{\frac{p_{k}-2}{2}}\right] \\
&=\sum_{k=1}^{m} c_{k}\left(b_{k}+r\right)^{\frac{p_{k}-4}{2}}\left[\left(p_{k}-2\right) r+b_{k}+r\right]=\sum_{k=1}^{m} c_{k}\left(b_{k}+r\right)^{\frac{p_{k}-4}{2}}\left[\left(p_{k}-1\right) r+b_{k}\right] .
\end{aligned}
$$

Therefore

$$
\begin{aligned}
\beta \Phi^{\prime}(r)-\Lambda(r) & =\beta \sum_{k=1}^{m} c_{k}\left(b_{k}+r\right)^{\frac{p_{k}-2}{2}}-\sum_{k=1}^{m} c_{k}\left(b_{k}+r\right)^{\frac{p_{k}-4}{2}}\left[\left(p_{k}-1\right) r+b_{k}\right] \\
& =\sum_{k=1}^{m} c_{k}\left(b_{k}+r\right)^{\frac{p_{k}-4}{2}}\left[\beta\left(b_{k}+r\right)-\left(p_{k}-1\right) r-b_{k}\right] \\
& =\sum_{k=1}^{m} c_{k}\left(b_{k}+r\right)^{\frac{p_{k}-4}{2}}\left[(\beta-1) b_{k}+\left(\beta-p_{k}+1\right) r\right] .
\end{aligned}
$$

Also, by (1.19), we write $g=g(\nabla u, u)$, referring to $\zeta \in \mathbb{R}^{n}$ as the variable corresponding to $\nabla u$ and to $\eta \in \mathbb{R}$ as the variable corresponding to $u$. By (1.20), we know that

$$
g_{\eta} \geq 0 \text {. }
$$

Moreover, by the homogeneity of $g$ in (1.21), we have that

$$
\nabla_{\zeta} g(\zeta, \eta) \cdot \zeta=\beta g(\zeta, \eta)
$$

As a consequence, by (1.14), (5.2) and (5.3) (and using short notations whenever possible), we have

$$
\mathscr{R}=-\frac{2 f g|\nabla u|^{2}}{\Phi^{\prime}}+2|\nabla u|^{4} g_{\eta}+\frac{2 f|\nabla u|^{2}}{\Lambda} \nabla_{\zeta} g \cdot \nabla u
$$




$$
\begin{aligned}
& \geq-\frac{2 f g|\nabla u|^{2}}{\Phi^{\prime}}+\frac{2 \beta f g|\nabla u|^{2}}{\Lambda} \\
& =\frac{2 f g|\nabla u|^{2}}{\Lambda \Phi^{\prime}}\left(\beta \Phi^{\prime}-\Lambda\right) .
\end{aligned}
$$

Hence, recalling (5.1), we obtain

$$
\mathscr{R} \geq \frac{2 f g|\nabla u|^{2}}{\Lambda \Phi^{\prime}} \sum_{k=1}^{m} c_{k}\left(b_{k}+|\nabla u|^{2}\right)^{\frac{p_{k}-4}{2}}\left[(\beta-1) b_{k}+\left(\beta-p_{k}+1\right)|\nabla u|^{2}\right] .
$$

Now we claim that

$$
\Xi:=f g \sum_{k=1}^{m} c_{k}\left(b_{k}+|\nabla u|^{2}\right)^{\frac{p_{k}-4}{2}}\left[(\beta-1) b_{k}+\left(\beta-p_{k}+1\right)|\nabla u|^{2}\right] \geq 0 .
$$

To prove (5.5) we distinguish six cases, according to the different assumptions in (1.22)-(1.27). To start with, let us assume that (1.22) is satisfied. Then, we have that

$$
\Xi=f g c_{1}\left(b_{1}+|\nabla u|^{2}\right)^{\frac{p_{1}-4}{2}}\left[(\beta-1) b_{1}+\left(\beta-p_{1}+1\right)|\nabla u|^{2}\right]=f g c_{1}\left(b_{1}+|\nabla u|^{2}\right)^{\frac{p_{1}-4}{2}}\left(p_{1}-2\right) b_{1} \geq 0,
$$

and this proves (5.5) in this case. The same way can be used to discuss cases (1.23) and (1.24), we omit them here.

If instead (1.25) is satisfied, we find that $(\beta-1) b_{k}+\left(\beta-p_{k}+1\right)|\nabla u|^{2} \geq 0$ and consequently $\Xi \geq 0$, which shows (5.5) in this case.

In addition, if (1.26) is satisfied, we see that

$$
\beta-p_{k}+1 \leq \beta-p_{1}+1 \leq 0,
$$

and thus

$$
\Xi=f g \sum_{k=1}^{m} c_{k}|\nabla u|^{p_{k}-4}\left(\beta-p_{k}+1\right)|\nabla u|^{2} \geq 0 .
$$

Finally, if (1.27) holds true, we see that

$$
\Xi=f g c_{1}\left(b_{1}+|\nabla u|^{2}\right)^{\frac{p_{1}-4}{2}}\left[(\beta-1) b_{1}+\left(\beta-p_{1}+1\right)|\nabla u|^{2}\right]=0 .
$$

This completes the proof of (5.5).

Then, the desired result follows from (5.4) and (5.5).

With the previous work, we can now establish Corollary 1.4, which gives a series of concrete situations in which our main gradient estimate holds true.

Proof of Corollary 1.4. By either (1.28) or (1.29) we have the validity of either (1.11) or (1.12) and consequently, by Proposition 1.1, we deduce that either Assumption A or Assumption B is satisfied.

This is one of the cornerstones to apply Theorem 1.2. The other fundamental ingredient to apply Theorem 1.2 lies in the reminder estimate (1.17), which we are now going to check. To this end, we want to exploit Proposition 1.3 and, for this, we need to verify that its assumptions are fulfilled in our setting. Indeed, we have that (1.20) and (1.21) follow from (1.30) and (1.31). Furthermore, at least one among (1.22)-(1.27) is satisfied, in light of (1.32)-(1.37). Condition 1.19 is also fulfilled, due to the structure of $g$ in (1.38). Therefore, all the hypotheses of Proposition 1.3 are satisfied, and consequently we deduce from Proposition 1.3 that $\mathscr{R} \geq 0$.

This in turn gives that condition (1.17) is satisfied and, as a consequence, we are in the position of exploiting Theorem 1.2. In this way, the desired result in (1.39) plainly follows from (1.18). 


\section{Proof of Theorem 1.7}

In this section, we prove Theorem 1.7. After our preliminary work, this part follows closely some arguments in CGS94, FV13. We provide full details in the specific case in which we are interested, for the facility of the reader.

Proof of Theorem 1.7. We take $x_{0}$ and $r_{0}$ as in the statement of Theorem 1.7 and we define

$$
\mathcal{V}:=\left\{x \in \mathbb{R}^{n} \text { s.t. } u(x)=r_{0}\right\} .
$$

Notice that $x_{0} \in \mathcal{V}$, and hence $\mathcal{V} \neq \varnothing$. Furthermore, by the continuity of $u$, we have that $\mathcal{V}$ is closed. We claim that

$$
\mathcal{V} \text { is also open. }
$$

From this, we would obtain that $\mathcal{V}=\mathbb{R}^{n}$, which is the thesis of Theorem 1.7. Therefore we focus on the proof of (6.1). For this, we fix $\hat{y} \in \mathcal{V}$ and $\hat{w} \in S^{n-1}$. For any $t \in \mathbb{R}$, we define

$$
\varphi(t):=u(\hat{y}+t \hat{w})-u\left(x_{0}\right) .
$$

We claim that there exist positive constants $c$ and $C$ such that

$$
\left|\varphi^{\prime}(t)\right| \leq C|\varphi(t)|, \quad \text { for all } t \in(-c, c)
$$

For this, we define

$$
\hat{p}:= \begin{cases}p & \text { if Assumption A holds with } p>2 \\ 2 & \text { otherwise }\end{cases}
$$

We also make use of the function $\Psi$ introduced in the proof of Lemma [3.1, which satisfies the functional identity

$$
\Psi^{-1}(r)=2 r \Phi^{\prime}(r)-\Phi(r), \quad \text { for all } r \in[0,+\infty)
$$

Let also

$$
G(r):=\Psi^{-1}(r)-\epsilon r^{\hat{p} / 2} .
$$

The parameter $\epsilon>0$ will be chosen conveniently small with respect to $M:=\|u\|_{W^{1, \infty}\left(\mathbb{R}^{n}\right)}$ and to the structural constants given in either (1.5) or (1.7). Observe that if $M=0$, then $u=0$ in $\mathbb{R}^{n}$ and so the result is true.

Now we take $r \in\left(0, M^{2}\right]$, with $M>0$, and $\sigma:=(\sqrt{r}, 0, \ldots, 0) \in \mathbb{R}^{n}$ and we use (1.13) and (1.3), and either (1.5) or (1.7), to see that

$$
\begin{aligned}
\Lambda(r) & =2 r \Phi^{\prime \prime}(r)+\Phi^{\prime}(r) \\
& =|\sigma|^{-2} \sum_{i, j} a_{i j}(\sigma) \sigma_{i} \sigma_{j} \\
& \geq\left\{\begin{array}{lr}
C_{1}(a+|\sigma|)^{p-2} & \text { if Assumption A holds and } p>2, \\
\frac{C_{1}}{(a+|\sigma|)^{2-p}} & \text { if Assumption A holds and } p \in(1,2], \\
\frac{C_{1}}{1+|\sigma|} & \text { if Assumption B holds }
\end{array}\right. \\
\geq & \left\{\begin{array}{lr}
\frac{C_{1}|\sigma|^{p-2}}{C_{1}} & \text { if Assumption A holds and } p>2, \\
\frac{C_{1}}{(a+M)^{2-p}} & \text { if Assumption A holds and } p \in(1,2], \\
\frac{1+M}{4} & \text { if Assumption B holds }
\end{array}\right.
\end{aligned}
$$




$$
\begin{aligned}
& \geq \begin{cases}\frac{\epsilon p}{2} r^{p / 2-1} \quad \text { if Assumption A holds and } p>2, \\
\epsilon \quad \text { if Assumption A holds and } p \in(1,2], \\
\epsilon \quad \text { if Assumption B holds }\end{cases} \\
& =\frac{\epsilon \hat{p}}{2} r^{\hat{p} / 2-1},
\end{aligned}
$$

as long as $\epsilon$ is small enough.

Furthermore, notice that, by $\Phi(0)=0$ and either (1.4) or (1.6), we have that $G(0)=0$. Also, by (1.13), we have

$$
G^{\prime}(r):=\Lambda(r)-\frac{\epsilon \hat{p}}{2} r^{\hat{p} / 2-1}
$$

for any $r>0$ and therefore $G^{\prime}(r) \geq 0$ for any $r \in\left(0, M^{2}\right]$, thanks to (6.4) (as long as $\epsilon$ is small enough). As a consequence, $G(r) \geq 0$ and therefore

$$
\Psi^{-1}(r) \geq \epsilon r^{\hat{p} / 2}
$$

for any $r \in\left(0, M^{2}\right]$. By taking $r:=|\nabla u(\hat{y}+t \hat{w})|^{2}$ in (6.5) and using (1.18), we obtain

$$
\begin{aligned}
\left|\varphi^{\prime}(t)\right|^{\hat{p}} & \leq|\nabla u(\hat{y}+t \hat{w})|^{\hat{p}} \\
& \leq \frac{1}{\epsilon} \Psi^{-1}\left(|\nabla u(\hat{y}+t \hat{w})|^{2}\right) \\
& \leq \frac{2}{\epsilon} F(u(\hat{y}+t \hat{w})) \\
& =\frac{2}{\epsilon}\left[F(u(\hat{y}+t \hat{w}))-F\left(u\left(x_{0}\right)\right)\right] .
\end{aligned}
$$

Now, we claim that if $r$ is sufficiently close to $r_{0}$ then there exists $C_{0}>0$ such that

$$
\left|F(r)-F\left(r_{0}\right)\right| \leq C_{0}\left|r-r_{0}\right|^{\hat{p}} .
$$

To check this we distinguish two cases, according to the value of $\hat{p}$. First of all, if $\hat{p}=2$, we use a second order Taylor expansion of $F$, and we conclude that

$$
\left|F(r)-F\left(r_{0}\right)\right| \leq \sup _{\rho \in\left[r_{0}-1, r_{0}+1\right]}\left|F^{\prime \prime}(\rho)\right|\left|r-r_{0}\right|^{2},
$$

from which (6.7) plainly follows in this case.

If, on the other hand, $\hat{p} \neq 2$, then the setting in (6.3) gives that Assumption A holds true with $p=\hat{p}>2$. Then, in this case (6.7) follows from (1.45). The proof of (6.7) is therefore complete.

Now, plugging (6.7) into (6.6), we get that there exists $c>0$ small enough such that

$$
\left|\varphi^{\prime}(t)\right|^{\hat{p}} \leq \frac{2 C_{0}}{\epsilon}\left|u(\hat{y}+t \hat{w})-u\left(x_{0}\right)\right|^{\hat{p}}=\frac{2 C_{0}}{\epsilon}|\varphi(t)|^{\hat{p}}, \quad t \in(-c, c) .
$$

Taking $C=\left(\frac{2 C_{0}}{\epsilon}\right)^{1 / \hat{p}}$, we obtain (6.2), as desired.

From (6.2) we obtain that the function $t \mapsto|\varphi(t)|^{2} e^{-2 C t}$ is non-increasing for small $t$. Accordingly, $|\varphi(t)| \leq|\varphi(0)| e^{C t}=0$ for small $t$, that is $\varphi(t)$ vanishes identically (for small $t$, independently of $\hat{w}$ ). By varying $\hat{w}$, we obtain that $u$ is constant in a small neighborhood of $\hat{y}$. This proves (6.1) and thus Theorem [1.7. 


\section{Acknowledgments}

Cecilia Cavaterra has been partially supported by GNAMPA (Gruppo Nazionale per l'Analisi Matematica, la Probabilitï $i \frac{1}{2}$ e le loro Applicazioni) of INdAM (Istituto Nazionale di Alta Matematica).

Serena Dipierro has been supported by the DECRA Project DE180100957 "PDEs, free boundaries and applications".

Serena Dipierro and Enrico Valdinoci have been supported by the Australian Research Council Discovery Project DP170104880 "N.E.W. Nonlocal Equations at Work".

$\mathrm{Zu}$ Gao has been supported by the Chinese Scholarship Council. This work was written on the occasion of a very pleasant and fruitful visit of Zu Gao at the Università di Milano, which we thank for the warm hospitality.

\section{References}

[AM01] Emilio Acerbi and Giuseppe Mingione, Regularity results for a class of functionals with non-standard growth, Arch. Ration. Mech. Anal. 156 (2001), no. 2, 121-140, DOI 10.1007/s002050100117. MR1814973

[And15] Ben Andrews, Moduli of continuity, isoperimetric profiles, and multi-point estimates in geometric heat equations, Surveys in differential geometry 2014. Regularity and evolution of nonlinear equations, Surv. Differ. Geom., vol. 19, Int. Press, Somerville, MA, 2015, pp. 1-47, DOI 10.4310/SDG.2014.v19.n1.a1. MR3381494

[AC13] Ben Andrews and Julie Clutterbuck, Sharp modulus of continuity for parabolic equations on manifolds and lower bounds for the first eigenvalue, Anal. PDE 6 (2013), no. 5, 1013-1024, DOI 10.2140/apde.2013.6.1013. MR3125548

[AX18] Ben Andrews and Changwei Xiong, Gradient estimates via two-point functions for elliptic equations on manifolds, arXiv e-prints (2018), arXiv:1808.09615, available at 1808.09615 .

[BG13] Agnid Banerjee and Nicola Garofalo, Gradient bounds and monotonicity of the energy for some nonlinear singular diffusion equations, Indiana Univ. Math. J. 62 (2013), no. 2, 699-736, DOI 10.1512/iumj.2013.62.4969. MR3158523

[BG15a] - Modica type gradient estimates for an inhomogeneous variant of the normalized p-Laplacian evolution, Nonlinear Anal. 121 (2015), 458-468, DOI 10.1016/j.na.2015.02.003. MR3348935

[BG15b] — Modica type gradient estimates for reaction-diffusion equations, Geometric methods in PDE's, Springer INdAM Ser., vol. 13, Springer, Cham, 2015, pp. 215-242. MR3587074

[BCM18] Paolo Baroni, Maria Colombo, and Giuseppe Mingione, Regularity for general functionals with double phase, Calc. Var. Partial Differential Equations 57 (2018), no. 2, Art. 62, 48, DOI 10.1007/s00526-018-1332-z. MR3775180

[Ber27] Serge Bernstein, Über ein geometrisches Theorem und seine Anwendung auf die partiellen Differentialgleichungen vom elliptischen Typus, Math. Z. 26 (1927), no. 1, 551-558, DOI 10.1007/BF01475472 (German). MR1544873

[CGS94] Luis Caffarelli, Nicola Garofalo, and Fausto Segàla, A gradient bound for entire solutions of quasilinear equations and its consequences, Comm. Pure Appl. Math. 47 (1994), no. 11, 1457-1473, DOI 10.1002/cpa.3160471103. MR1296785

[CFV12] Diego Castellaneta, Alberto Farina, and Enrico Valdinoci, A pointwise gradient estimate for solutions of singular and degenerate PDE's in possibly unbounded domains with nonnegative mean curvature, Commun. Pure Appl. Anal. 11 (2012), no. 5, 1983-2003, DOI 10.3934/cpaa.2012.11.1983. MR2911121

[CY75] S. Y. Cheng and S. T. Yau, Differential equations on Riemannian manifolds and their geometric applications, Comm. Pure Appl. Math. 28 (1975), no. 3, 333-354, DOI 10.1002/cpa.3160280303. MR0385749

[CFV14] Matteo Cozzi, Alberto Farina, and Enrico Valdinoci, Gradient bounds and rigidity results for singular, degenerate, anisotropic partial differential equations, Comm. Math. Phys. 331 (2014), no. 1, 189-214, DOI 10.1007/s00220-014-2107-9. MR3231999 
[CMM17] Giovanni Cupini, Paolo Marcellini, and Elvira Mascolo, Regularity of minimizers under limit growth conditions, Nonlinear Anal. 153 (2017), 294-310, DOI 10.1016/j.na.2016.06.002. MR3614673

[DG02] Donatella Danielli and Nicola Garofalo, Properties of entire solutions of non-uniformly elliptic equations arising in geometry and in phase transitions, Calc. Var. Partial Differential Equations 15 (2002), no. 4, 451-491, DOI 10.1007/s005260100133. MR1942128

[FV10a] Alberto Farina and Enrico Valdinoci, Flattening results for elliptic PDEs in unbounded domains with applications to overdetermined problems, Arch. Ration. Mech. Anal. 195 (2010), no. 3, 1025-1058, DOI 10.1007/s00205-009-0227-8. MR2591980

[FV10b] _ A pointwise gradient estimate in possibly unbounded domains with nonnegative mean curvature, Adv. Math. 225 (2010), no. 5, 2808-2827, DOI 10.1016/j.aim.2010.05.008. MR2680184

[FV11] _ A pointwise gradient bound for elliptic equations on compact manifolds with nonnegative Ricci curvature, Discrete Contin. Dyn. Syst. 30 (2011), no. 4, 1139-1144, DOI 10.3934/dcds.2011.30.1139. MR2812957

[FV13a] _ Pointwise estimates and rigidity results for entire solutions of nonlinear elliptic PDE's, ESAIM Control Optim. Calc. Var. 19 (2013), no. 2, 616-627, DOI 10.1051/cocv/2012024. MR3049726

[FV13b] _ On partially and globally overdetermined problems of elliptic type, Amer. J. Math. 135 (2013), no. 6, 1699-1726, DOI 10.1353/ajm.2013.0052. MR3145008

[FV14] _ Gradient bounds for anisotropic partial differential equations, Calc. Var. Partial Differential Equations 49 (2014), no. 3-4, 923-936, DOI 10.1007/s00526-013-0605-9. MR3168616

[Gar09] Nicola Garofalo, Gradient bounds for the horizontal p-Laplacian on a Carnot group and some applications, Manuscripta Math. 130 (2009), no. 3, 375-385, DOI 10.1007/s00229-009-0294-z. MR2545524

[GL89] Nicola Garofalo and John L. Lewis, A symmetry result related to some overdetermined boundary value problems, Amer. J. Math. 111 (1989), no. 1, 9-33, DOI 10.2307/2374477. MR980297

[GS99] Nicola Garofalo and Elena Sartori, Symmetry in exterior boundary value problems for quasilinear elliptic equations via blow-up and a priori estimates, Adv. Differential Equations 4 (1999), no. 2, 137-161. MR1674355

[GT83] D. Gilbarg and N.S. Trudinger, Elliptic Partial Differential Equations of Second Order, 2nd ed, Comprehensive Studies in Mathematics, vol. 224, Springer-Verlag, Berlin, 1983. MR0737190

[Ham93] Richard S. Hamilton, A matrix Harnack estimate for the heat equation, Comm. Anal. Geom. 1 (1993), no. 1, 113-126, DOI 10.4310/CAG.1993.v1.n1.a6. MR1230276

[Mod85] Luciano Modica, A gradient bound and a Liouville theorem for nonlinear Poisson equations, Comm. Pure Appl. Math. 38 (1985), no. 5, 679-684, DOI 10.1002/cpa.3160380515. MR803255

[Pay76] L. E. Payne, Some remarks on maximum principles, J. Analyse Math. 30 (1976), 421-433, DOI 10.1007/BF02786729. MR0454338

[PP80] L. E. Payne and G. A. Philippin, On maximum principles for a class of nonlinear second-order elliptic equations, J. Differential Equations 37 (1980), no. 1, 39-48, DOI 10.1016/0022-0396(80)90086-8. MR583337

[PQS07] Peter Poláčik, Pavol Quittner, and Philippe Souplet, Singularity and decay estimates in superlinear problems via Liouville-type theorems. II. Parabolic equations, Indiana Univ. Math. J. 56 (2007), no. 2, 879-908, DOI 10.1512/iumj.2007.56.2911. MR2317549

[PS07] Patrizia Pucci and James Serrin, The maximum principle, Progress in Nonlinear Differential Equations and their Applications, vol. 73, Birkhäuser Verlag, Basel, 2007. MR2356201

[SZ06] Philippe Souplet and Qi S. Zhang, Sharp gradient estimate and Yau's Liouville theorem for the heat equation on noncompact manifolds, Bull. London Math. Soc. 38 (2006), no. 6, 1045-1053, DOI 10.1112/S0024609306018947. MR2285258

[Spe81] René P. Sperb, Maximum principles and their applications, Mathematics in Science and Engineering, vol. 157, Academic Press, Inc. [Harcourt Brace Jovanovich, Publishers], New York-London, 1981. MR615561 